\title{
Reorganization of Somatosensory Cortical Areas $3 b$ and 1 after Unilateral Section of Dorsal Columns of the Spinal Cord in Squirrel Monkeys
}

\author{
Hui-Xin Qi, ${ }^{1}$ Li M. Chen, ${ }^{2,3}$ and Jon H. Kaas ${ }^{1,2}$ \\ Departments of ${ }^{1}$ Psychology, and ${ }^{2}$ Radiology and Radiological Science, and ${ }^{3}$ Institute of Imaging Science, Vanderbilt University, Nashville, Tennessee \\ 37240
}

An incomplete lesion of the ascending afferents from the hand in the dorsal columns of the spinal cord in monkeys is followed after weeks of recovery by a reactivation of much of the territory of the hand representations in primary somatosensory cortex (area 3b). However, the relationship between the extent of the dorsal column lesion and the amount of cortical reactivation has not been clear. Largely, this is due to the uncertainties about axon sparing after spinal cord lesions. Here, we unilaterally sectioned dorsal column afferents in the cervical spinal cord (C4-C6) in adult squirrel monkeys. After weeks of recovery, cholera toxin subunit B (CTB) was injected into the distal phalanges to label normal and surviving afferents to the cuneate nuclei representing the hands. Days later, the responsiveness of neurons in cortical areas $3 \mathrm{~b}$ and 1 to tactile stimulation on the hand was evaluated in a microelectrode mapping session. The sizes and densities of CTB-labeled patches in the cuneate nuclei of both sides were quantified and compared. The results indicate that extensive reactivations of the hand representations in cortical areas $3 \mathrm{~b}$ and 1 occur contralateral to the spinal cord lesion, even when $<1 \%$ of labeled dorsal column terminations in the cuneate nucleus remained. These results raise the possibilities that secondary afferents from innervated neurons in the spinal cord contribute to the reactivation, and that the reactivation of area 1 is not completely dependent on inputs from area $3 b$.

\section{Introduction}

After partial or nearly complete damage to the nervous system, mature humans and other mammals typically demonstrate some behavioral recovery over a period of days to months (Almli and Finger, 1988; Nudo et al., 2000; Muellbacher and Hallett, 2006; Kaas et al., 2008). The reasons for these recoveries are not completely clear, but there is evidence that the potentiation of existing neuronal connections, as well as the growth of new synaptic connections, play important roles in the process (Garraghty et al., 1991; Florence and Kaas, 1995; Jain et al., 2000; Darian-Smith and Ciferri, 2005; Qi et al., 2010). One approach to studying recovery after damage to the nervous system in monkeys is to evaluate the responsiveness of somatosensory cortex to tactile stimuli after weeks of recovery from a sensory loss. A useful way of providing an incomplete sensory loss is sectioning the ascending branches of peripheral afferents as they ascend in the dorsal

Received May 11, 2011; revised July 9, 2011; accepted Aug. 1, 2011.

Author contributions: H.-X.Q., L.M.C., and J.H.K. designed research; H.-X.Q. and L.M.C. performed research; H.-X.Q. analyzed data; H.-X.Q., L.M.C., and J.H.K. wrote the paper.

This research was supported by NIH Grant NS16446, and Dana and Christopher Reeve Foundation grant to J.H.K. NIH Grants NS057399 and NS067017 to H.-X.Q.; and NIH Grant DE024831 and a Dana Foundation grant to L.M.C. We thank Laura Trice and Chaohui Tang for technical support; Christina M. Cerkevich and Dr. Barbara Dillenburger for data collection; Gabriella E. DiCarlo for data analysis; and Pooja Balaram and Dr. Jamie L. Reed for comments on the manuscript. We also thank Dr. Anna Roe for use of her laboratory facility.

The authors declare no competing financial interests.

Correspondence should be addressed to Hui-Xin Qi, Vanderbilt University, Department of Psychology, 301 Wilson Hall, 111 21st Avenue South, Nashville, TN 37240. E-mail: huixin.qi@vanderbilt.edu.

DOI:10.1523/JNEUROSCI.2366-11.2011

Copyright $\odot 2011$ the authors $\quad 0270-6474 / 11 / 3113662-14 \$ 15.00 / 0$ columns of the cervical spinal cord. Other branches of the afferents terminate in the dorsal horn of the spinal cord, and these neurons provide sensory information to spinal cord circuits, as well as contribute to other ascending sensory pathways (Willis and Coggeshall, 2004). Thus, locomotion and other motor behaviors largely appear to be normal after such lesions, while grasping and food retrieval behavior are impaired initially, but often recover over a few postlesion weeks (Leonard et al., 1992; Cooper et al., 1993; Jain et al., 1997). After unilateral dorsal column lesions at a high cervical spinal cord level, the contralateral primary somatosensory cortex (area $3 \mathrm{~b}$ ) can become completely unresponsive to tactile stimuli on the hand and other parts of the lower body, or islands of cortex can remain responsive to preserved dorsal columns afferents after incomplete lesions (Jain et al., 1997, 2000, 2008). After months of recovery, most or all of the cortex in area $3 \mathrm{~b}$ can recover responsiveness to preserved sensory inputs. This suggests that preserved inputs from the hand become potentiated by reduced inhibition (Garraghty et al., 1991, 2006; Jones, 1993) and the formation of new connections (e.g., Florence and Kaas, 1995; Jain et al., 2000; Churchill et al., 2004). Another possibility is that neurons in the dorsal horn of the spinal cord with inputs from the hand project to the cuneate nucleus and contribute to the recovery (Rustioni et al., 1979; Bennett et al., 1983; Willis and Coggeshall, 2004). Although these secondorder afferents are thought to join the dorsal columns before they reach the cuneate nucleus, some of them possibly avoid the lesion zone and reach the cuneate nucleus. Finally, spinothalamic neurons may contribute to cortical reactivations. 
Here, we relate the extents of afferent loss to the extents and types of cortical reactivations by afferents from the hand after 7-9 weeks of recovery from large to nearly complete dorsal column lesions in squirrel monkeys (SMs). The results indicate that extensive amounts of deprived cortex can be reactivated, even when very few dorsal column afferents from the hand remain.

\section{Materials and Methods}

The somatotopic organization of the hand representations in somatosensory areas $3 \mathrm{~b}$ and 1 were studied in four adult male squirrel monkeys (Saimiri bolivians). Three monkeys received a unilateral section of the dorsal columns of the spinal cord at a C4-C6 cervical level. The fourth monkey received a unilateral small lesion in the white matter near the lateroventral fasciculus of the spinal cord at cervical C5 level without damaging the dorsal columns. The hand regions of areas $3 \mathrm{~b}$ and 1 contralateral to the spinal cord lesion were mapped with microelectrodes. Areas $3 \mathrm{~b}$ and 1 ipsilateral to the spinal cord lesions were also mapped to serve as controls.

Before surgery, the monkeys were trained and tested on reaching for food to evaluate hand use. In addition, the somatotopy of the hand representations in cortical areas $3 \mathrm{~b}$ and 1 were evaluated noninvasively with functional magnetic resonance imaging (fMRI) before, and several times after, sectioning the contralateral dorsal columns of the spinal cord. Seven to 9 weeks after the effective spinal cord lesions, the digits of both hands were injected with a tracer [cholera toxin subunit B (CTB)] so that transport of the tracer to the cuneate nuclei of both sides could be evaluated as a measure of the effectiveness of the spinal cord lesion in sectioning ascending afferents to the dorsal column nuclei. Six days later, the monkeys were anesthetized, and the responsiveness and somatotopy of the hand representations in cortical areas $3 \mathrm{~b}$ and 1 were determined by recording from neurons with single microelectrodes. This microelectrode mapping was followed by an optical imaging session to further evaluate the responsiveness and somatotopy of the hand region of areas $3 \mathrm{~b}$ and 1 . Upon the completion of these procedures, the monkeys were given a lethal dose of anesthetic and perfused with fixative. The brains and spinal cords were histologically processed, and the results were analyzed. All experimental procedures were approved by the Vanderbilt University Animal Care and Use Committee, and followed the guidelines of the National Institute of Health Guides for the Care and Use of Laboratory Animals.

Dorsal column section. In preparation for the dorsal column section of the spinal cord, each monkey was initially anesthetized with ketamine hydrochloride $(15 \mathrm{mg} / \mathrm{kg}$, i.m.) and then maintained in a stereotaxic head holder at a surgical level of anesthesia with $1-3 \%$ isoflurane. The depth of anesthesia was monitored by recording the heart and respiration rates, and testing for withdrawal reflexes. Rectal body temperature was maintained at $37-38^{\circ} \mathrm{C}$. Under aseptic conditions, a portion of the cervical spinal cord was exposed, and the dorsal columns were sectioned on one side with a pair of fine surgical scissors at cervical level C4-C6. Dura was replaced with Gelfilm and covered with Gelfoam. The opening was closed, and the skin sutured. The monkeys were carefully monitored until they were fully recovered from anesthesia and then returned to their home cage. Monkeys received antibiotics and analgesics for $2-3 \mathrm{~d}$ after surgery. Animals' cage behavior and food intake typically returned to normal shortly after surgery. Further details about surgical procedures can be found in previous publications from the laboratory (Jain et al., 1997, 2008).

Tracer injection in digits. To determine the levels of the spinal cord where the sensory inputs from the digits terminated in the dorsal horn, and the amounts of inputs from the digits to the cuneate nuclei, 10-20 $\mu \mathrm{l}$ of $1 \%$ CTB (Sigma) was subcutaneously injected into digits 1 (D1), 3 , and 5 of both hands of these experimental monkeys while they were anesthetized with 1-3\% isoflurane. Procedures followed those described previously (Florence et al., 1991; Qi and Kaas, 2006).

Microelectrode recording and mapping. The responsiveness of somatosensory cortical areas $3 b$ and 1 was determined 7-9 weeks after the extensive contralateral spinal cord lesions by recording neuronal responses with low-impedance microelectrodes (1-3 $\mathrm{M} \Omega$ at $1 \mathrm{kHz}$ ) from large numbers of recording sites across the hand representations in areas $3 \mathrm{~b}$ and 1. Cortex ipsilateral to the dorsal column lesion was also partially mapped to directly compare the responsiveness and the receptive field (RF) sizes of neurons in normal and deprived areas $3 \mathrm{~b}$ and 1 in the same animals. Additionally, we mapped the somatosensory cortex of a surgical control monkey that received a laminectomy at a cervical level, but the dorsal column was untouched.

The monkeys were first tranquilized with ketamine $(10 \mathrm{mg} / \mathrm{kg}$, i.m.) and xylazine $(0.4 \mathrm{mg} / \mathrm{kg}, \mathrm{i} . \mathrm{m}$.$) , and then anesthetized and maintained at the$ surgical level with isoflurane $(0.8-1.1 \%)$ delivered in a 70:30 $\mathrm{NO}_{2} / \mathrm{O}_{2}$ mixture. Somatosensory areas $3 \mathrm{~b}$ and 1 were exposed and covered with warm agar or silicone oil to prevent desiccation. The cortical surface was photographed, and the blood vessel pattern was used to guide electrode penetrations, which were marked on the photograph. Within and near the hand representations of areas $3 \mathrm{~b}$ and 1 (Sur et al., 1982), the microelectrode penetrations were placed densely in $\sim 300 \mu \mathrm{m}$ spacing with allowances for blood vessels. An effort was made to cover most or all of the hand representations in areas $3 \mathrm{~b}$ and 1 during the recording session. Magnitudes of neuronal responses to the tapping and manipulation of the hand, as indicated by the acoustic strength of neuron firing audio output, were constantly evaluated as the microelectrode passed through the superficial to the middle layers of cortex (up to $1000 \mu \mathrm{m}$ depth). For each penetration, we characterized neurons' receptive field location, size, and stimulus preference to light touch, tapping, and joint movement stimuli at the site where the strongest evoked response occurred. Neurons were classified as cutaneous if they responded to light contact on the skin or hair movement, high threshold if they required taps to the skin, and noncutaneous or "deep" if they responded only to the manipulation of joints and muscles. The minimum receptive field was determined by the area of skin where light touches with a cotton tipped probe activated the recorded neurons (Merzenich et al., 1978). When a receptive field was defined, it was outlined on a drawing of the hand or other relevant body part. At the end of the recording sessions, electrolytic marking lesions were placed at selected sites by passing current $(10 \mu \mathrm{A}, 10 \mathrm{~s})$ via the microelectrode. These marking lesions were used to relate the recording results to cortical architecture.

Tissue processing and histology. At the end of the terminal microelectrode mapping session, each monkey was given a lethal dose of anesthetic (sodium pentobarbital), and when areflexive, perfused transcardially with $0.9 \%$ PBS, $\mathrm{pH} 7.4$, followed by $2-4 \%$ paraformaldehyde in $\mathrm{PB}$, followed by $2-4 \%$ paraformaldehyde with $10 \%$ sucrose in $\mathrm{PB}$. The brain and spinal cord were removed separately. Cortex was separated from subcortical structures, manually flattened, and kept flat between glass slides (for progressive steps in flattening squirrel monkey cortex, see Gharbawie et al., 2011). The cortex, brainstem, and spinal cord were stored overnight in $30 \%$ sucrose in $\mathrm{PB}$ for cryoprotection. The cortex was cut parallel to the surface into $40 \mu \mathrm{m}$ sections on a freezing microtome, and the sections were stained for myelin (Gallyas, 1979) to reveal the cortical locations of the lesions tracks along electrode penetrations. The brainstem was sectioned in the coronal plane and the spinal cord in the horizontal plane, both at $40 \mu \mathrm{m}$. Every fourth section of the brainstem and every second section of the spinal cord was processed with immunohistochemistry to reveal CTB (Qi and Kaas, 2006). Another series of brainstem and spinal cord sections was processed for cytochrome oxidase (Wong-Riley, 1979) to reveal brainstem and spinal cord architecture (Qi and Kaas, 2006).

Data analysis. To reconstruct spinal cord lesions, images of spinal cord sections were acquired using a Nikon E800 microscope and a Nikon DXM1200 camera. All horizontally cut spinal cord sections were aligned along the midline, and a pinhole was made by a penetration perpendicular to the plane of the section. The maximal extent of the lesion, the white matter, and the gray matter were measured, and a coronal view of the lesion site was reconstructed from the sections with Adobe Illustrator software (Jain et al., 1997).

Representations of the hand and adjoining parts of the face and arm in cortical areas $3 \mathrm{~b}$ and 1 were reconstructed by relating receptive field locations to cortical recording sites transposed from their marked locations on photographs of the brain to cortical brain sections, which were cut parallel to the brain surface. The electrode penetrations and record- 
A

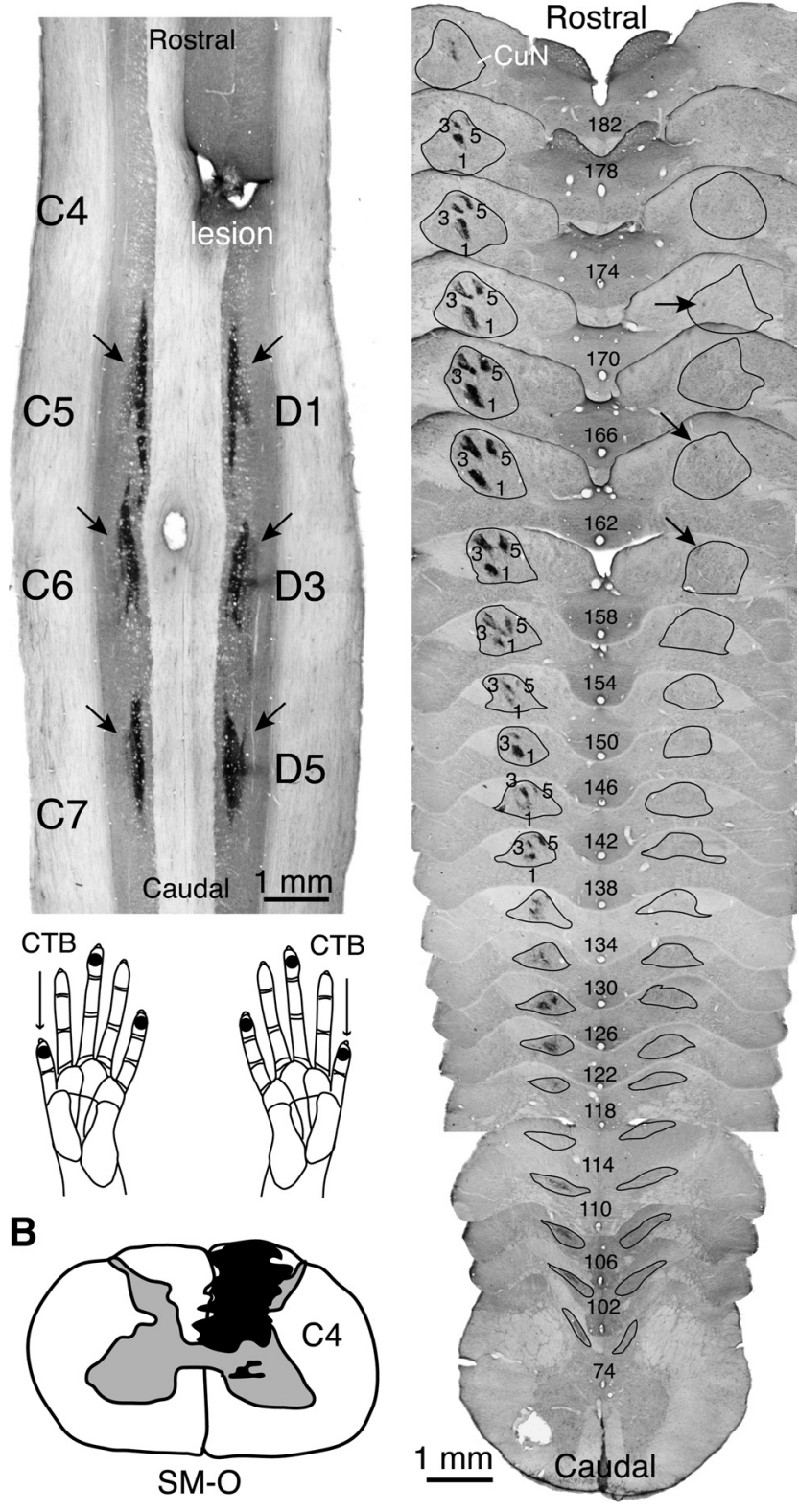

Figure 1. Terminations of peripheral afferents in the dorsal horn of the spinal cord and cuneate nucleus of the brainstem labeled by injections of (TB into the distal phalange of digits 1,3 , and 5 of a squirrel monkey (SM-0). $\boldsymbol{A}$, A horizontally cut CTB-immunoreacted section of the spinal cord showing the location of the lesion on the upper right and labeled terminal fields after tracer injections. Cervical segments 4-7 are indicated, and foci of labels from injections in digits 1, 3, and 5 are marked with arrows. Injection sites in the digits are indicated by black dots on the schematic drawings of hands on the bottom of $\boldsymbol{A}$. $B$, Transverse view of spinal cord through cervical segment $C 4-C 5$ with the reconstructed extent of the lesion in black. The lesion was reconstructed from a series of horizontally cut sections. C, A series of coronally cut CTB-immunoreacted sections through dorsal column nuclei of brainstem. The cuneate nucleus is outlined, and numbers 1,3 , and 5 mark foci of afferents labeled by injections in digits 1,3 , and 5 . Note that there are only a few detectable foci of axon fibers on the lesioned (right) side (arrows). The numbers in the section centers indicate the position of each section in the series. Note that not all sections are included. CuN, Cuneate nucleus of brainstem.

ing sites were located relative to electrolytic lesions used to mark recording sites, and blood vessels and other tissue landmarks that were apparent in both the brain section and the brain surface photographs. The known distances between microlesions were used to correct for the slight shrinkage of brain sections due to processing.
A
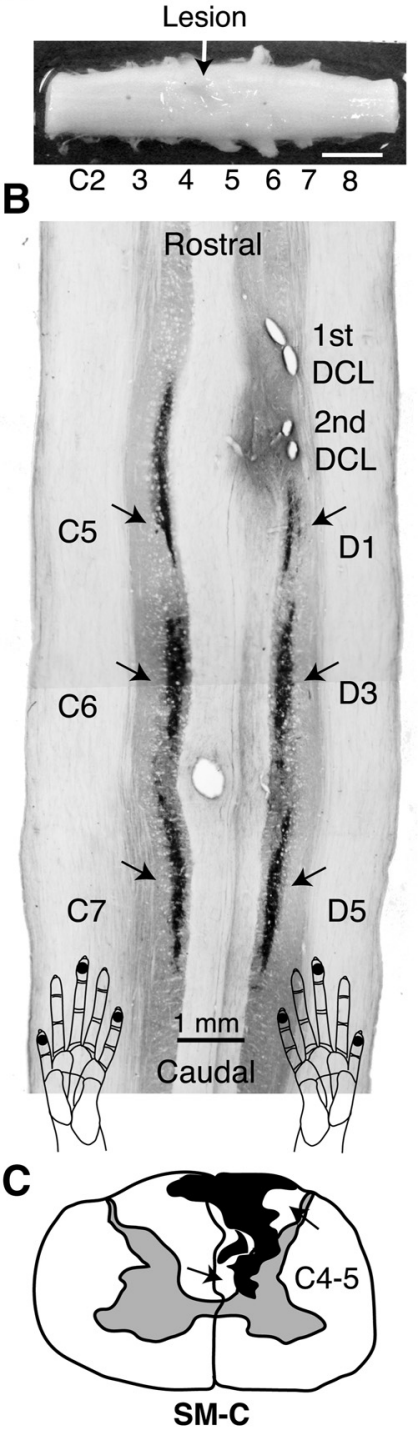

D

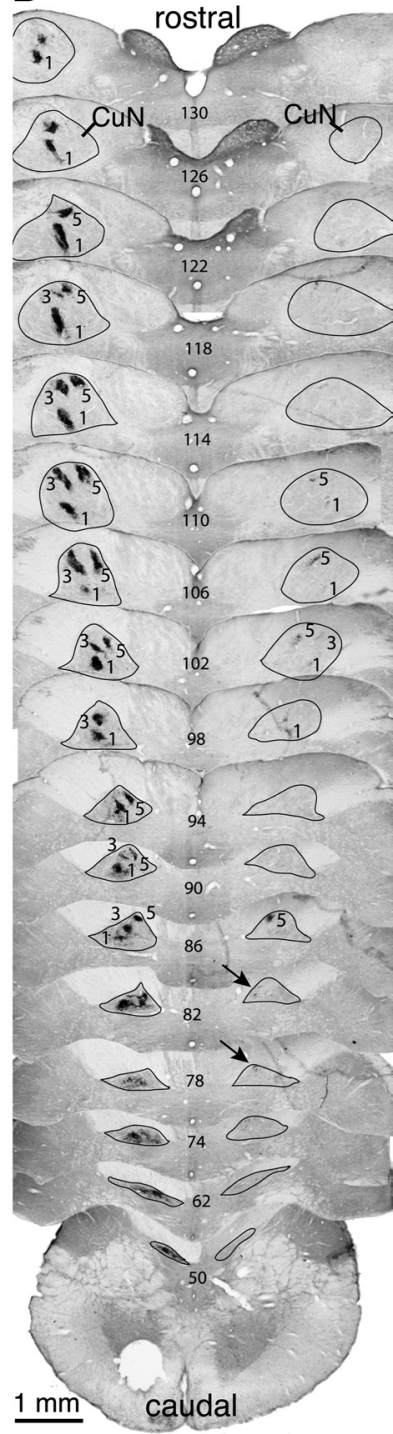

Figure 2. Terminations of peripheral afferents in the dorsal horn of the spinal cord and cuneate nucleus of SM-C. A, A portion of the cervical spinal cord through C2-C8. The arrow points to the lesion on the right side of the spinal cord. Scale bars, $5 \mathrm{~mm}$. B, Foci of peripheral afferents labeled in the dorsal horn of the spinal cord by injections of tracer CTB into the distal phalange of digits 1,3, and 5 of both hands. Traces of the first and second lesion are on the upper right. C, A transverse view of spinal cord through cervical segment $C 4-C 5$ indicating the extent of lesion (black). The lesion was reconstructed from a series of horizontally cut sections. The arrows mark the preserved zones in the dorsal columns. $\boldsymbol{D}$, A series of sections through the cuneate nucleus of the brainstem. The numbers in the section centers indicate the position of each section in the series. The cuneate nucleus is outlined, and numbers 1,3 , and 5 mark foci of afferents labeled by injections in digits 1,3 , and 5 . Only a few weakly labeled foci survived on the side of lesion (marked by arrows, or numbers). For other conventions, see Figure 1.

To quantitatively measure the distribution (areal size) and density (lightness) of CTB-labeled terminal fields in the dorsal horn of the spinal cord and in the cuneate nuclei of the brainstem, we used NIH ImageJ, version 144 software (available as freeware from http://rsbweb.nih.gov/ij/) (Tang et al., 2010). For areal measurement of foci of labeled terminals, digital photomicrographs of CTB-immunoreacted brainstem sections were acquired with a Sony digital camera attached to a Nikon E-800 microscope. Each image was then loaded into ImageJ and converted into an 8 bit image. Then, a threshold was set to a value of 133 for all three CTB injected cases by moving the bottom slider in the drop-down window (Image/Adjust/Threshold) in ImageJ software. The magic wand tool was used to outline the zones above threshold. The area and number of labeled pixels were obtained through the 

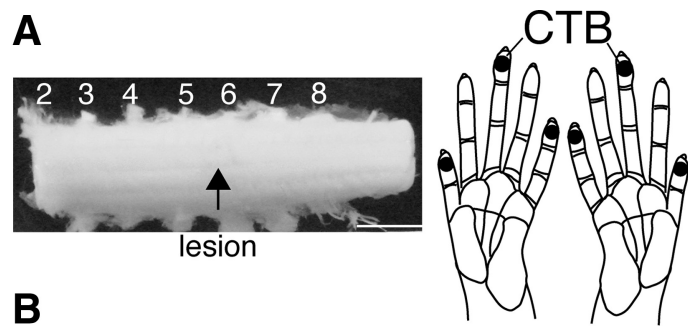

B
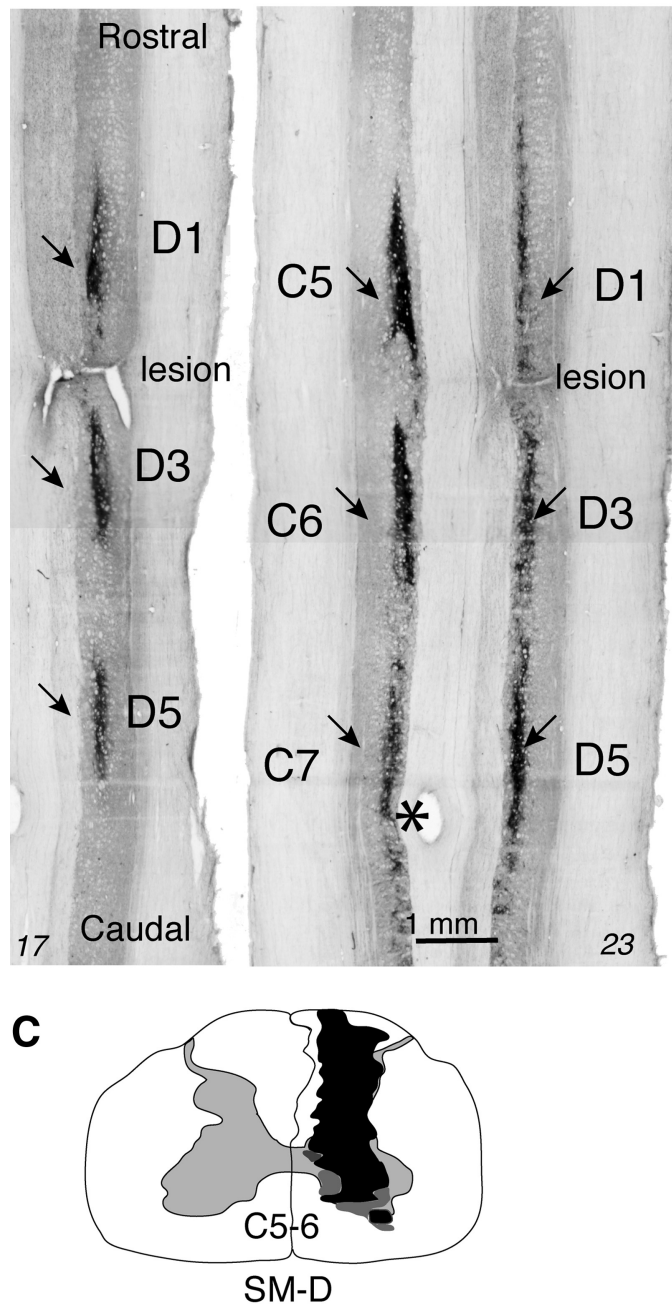
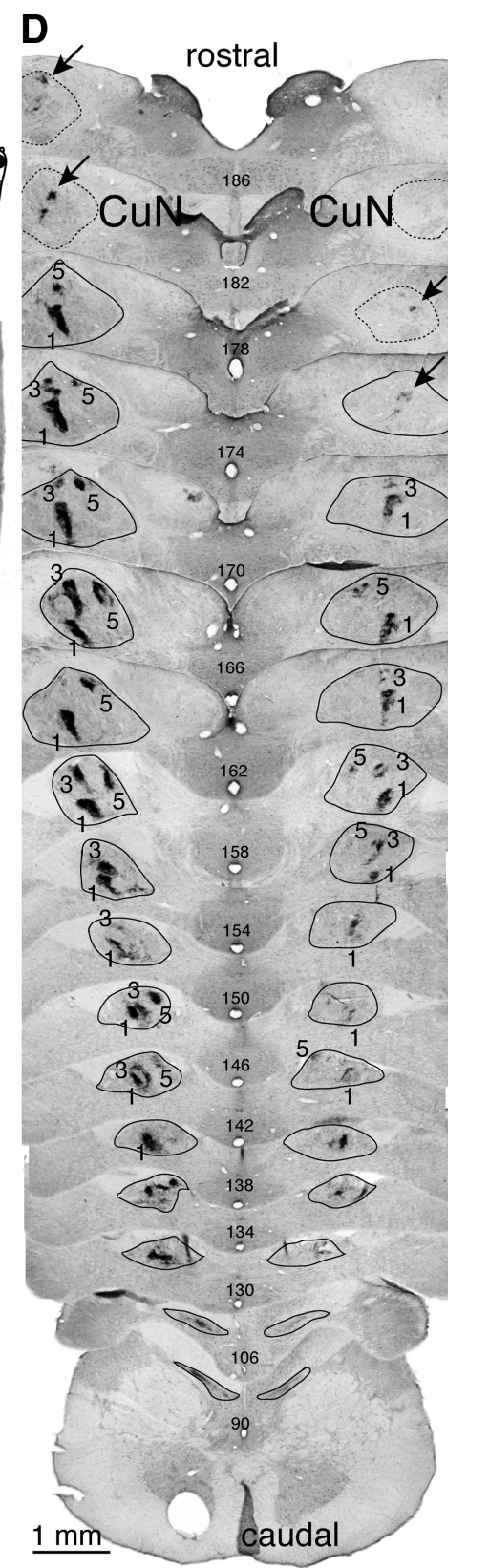

Figure 3. Terminations of peripheral afferents in the dorsal horn of the spinal cord and cuneate nucleus of SM-D.A, A portion of cervical spinal cord through C2-C8. The arrow points to the lesion on the right side of the spinal cord. Scale bar, $5 \mathrm{~mm}$. B, Foci of the peripheral afferent terminations in the dorsal horn of the spinal cord labeled by injections of tracer CTB into the distal phalange of digits 1,3, and 5. The dorsal column section was at cervical cord C5-C6 on the right side of the spinal cord, which is at the level of peripheral afferents from digit 2 . The right half of section 17 and both halves of section 23 are shown. C, Transverse view of spinal cord through cervical segment $\mathrm{C} 4-\mathrm{C} 5$ indicating the extent of lesion (black). The lesion was reconstructed from a series of horizontally cut sections. $\boldsymbol{D}$, A series of coronally cut CTB-immunoreacted sections through the dorsal column nuclei of the brainstem. The cuneate nucleus is outlined, and numbers 1,3 , and 5 mark labeled foci of afferents from digits 1,3, and 5 . More foci of axons are labeled on the lesioned right side in SM-D than in the other two monkeys, especially for D1 foci (see Figs. 1,2). However, the density of the labeled terminations in the D1 territory was reduced on the lesion side compared with those of the intact side. The numbers in the section centers indicate the position of each section in the series. For other conventions, see Figure 1.

for all three channels were then averaged [average reflectance $=($ red + green + blue $) / 3]$. The inversed average reflectance was used as the measure of label density in this study.

To statistically compare the extents (areal size) and densities of the CTB label between the intact side and lesioned side in the dorsal horn of the spinal cord and in the cuneate nuclei of the brainstem, the Wilcoxon matched-pairs signed-rank test was applied using GraphPad InStat software. All data are reported as the mean $\pm \mathrm{SD}$.

\section{Results}

Cortical recording results are based on a total of 569 microelectrode penetrations from three deprived cortices $(168,166,235)$, with an average of 190 penetrations per animal. For nondeprived cortex, the territories of only two to three digits were densely mapped, as our goal was to characterize the neuronal response properties, stimulus preference, and receptive field sizes. For the dorsal column section, we intended to make a lesion at the middle cervical level (C5) that would spare some ascending inputs from digit 1 , while potentially sectioning most or all inputs from digits 2-5. As the lesions were large, but incomplete, and varied somewhat in extent and spinal cord level, the results are described for each case, starting with the one with the most extensive spinal cord lesion. We describe the histological evaluation of the extents of lesions first, followed by electrophysiological recording and mapping results from areas $3 \mathrm{~b}$ and 1 . The results indicate that extensive but incomplete lesions of the ascending branches of peripheral nerve afferents of the spinal cord were followed after weeks of recovery by major reactivations of the hand representations in primary somatosensory cortex (area $3 \mathrm{~b}$ ) and adjoining area 1 . These observations demonstrate the importance of a few remaining first-order afferents, as well as possible second-order afferents, in the reactivation of somatosensory cortex and likely the behavioral recovery of the animal.

The extent and spinal cord levels of the unilateral lesions of the dorsal columns As our interpretations of the recording results from somatosensory cortex critically depend on independent estimations of the extents of the dorsal column lesions, we determined the effectiveness of these lesions in two ways. First, the extents of the

measure function. The set scale function in ImageJ was used to convert pixel numbers to standard measurement units. In one case (SM-D), we also measured label density. This procedure was performed by loading the RGB color image into ImageJ software. The labeled zones were outlined with the freehand selection tool, and the value of density (reflectance) was automatically calculated by the software with a plugin written by Wayne Rasband (http:// rsbweb.nih.gov/ij/plugins/rgb-measure.html).This plugin separately measures the red, green, and blue channels of an RGB image. The values obtained lesions were determined in the traditional way by outlining the locations of missing tissue and adjoining scar tissue on sections through the spinal cord. For the present cases with dorsal column lesions, this was done in sections cut in the horizontal plane along the length of the spinal cord. This proved to be the most useful plane for such reconstructions. Second, we compared the 
amounts and locations of patches of labeled axon terminals from both hands in the dorsal horn of the spinal cord, indicating the spinal cord levels where digit inputs enter and, in the cuneate nuclei, where dorsal column lesions would alter the amounts and locations of labeled axon terminals. These axons had been labeled by injections of CTB into digits 1,3 , and 5 of both hands.

\section{$S M-O$}

In this animal, CTB-immunoreacted, horizontally cut spinal cord sections revealed that the dorsal column lesion at the $\mathrm{C} 4$ level on the right side of the spinal cord was rostral to the sites where afferents from the digits enter the spinal cord. Foci of CTB-labeled axon terminals for D1, D3, and D5 were present in the dorsal horns of both sides below the lesion level (Fig. $1 \mathrm{~A}$ ). As shown in reconstructed transverse views of spinal cord in Figure $1 \mathrm{~B}$, the lesion was almost complete in the dorsal columns except for minor sparing on the dorsolateral corner near the dorsal root entrance zone. The ipsilateral dorsal horn and a portion of the intermediate zone were also damaged. In the cuneate nuclei, the pattern of the labeled axon terminals on the lesioned side (the right side of sections) was very different from that of the intact side (the left side). As shown in Figure $1 C$, three patches of labeled axon terminal fields from the three CTB-injected digits can be identified across many sections on the intact side, but there are only a few sparse patches of label apparent on the lesioned side (pointed out with arrows), indicating that the lesion was extensive and almost complete. By their locations, those sparse patches likely reflected preserved inputs from digits 3 and 5, as the normal locations of terminations from digits 1,3 , and 5 in the dorsal horn and cuneate nucleus are known from previous studies (Florence et al., 1989). We conclude that the extensive lesion in SM-O was rostral to inputs from all of the digits, and it cut nearly all of the ascending branches of afferents from the hand in the cuneate fasciculus. A sparse focus of label in a few sections of the cuneate nucleus indicated that some afferents from digits 3 and 5, and perhaps other parts of the hand, remained. Yet, the deafferentation of the cuneate nucleus was extensive. A quantitative evaluation of the effectiveness of the lesion in this and other cases is presented in a following section of the Results.

\section{$S M-C$}

In SM-C, two lesions of the dorsal columns were made to achieve an extensive but incomplete sectioning of the afferents of the cuneate fasciculus. The first lesion was placed at the C4 level, which was rostral to the entrance zone where most of the afferents from the digits terminate in the dorsal horn. After the lesion, the monkey failed to demonstrate the expected impairment of reaching and grasping behavior. As a result, nearly 10 weeks after the first lesion, a second lesion was placed at the C4-C5 junction $\sim 1$ $\mathrm{mm}$ caudal to the first lesion. A lesion at this level would spare some of the afferents from digit 1 . Postmortem examination of the spinal cord sections confirmed that the first lesion was small and involved only a portion of the lateral part of the cuneate fasciculus. The second lesion was more extensive, and the majority of the ascending afferents from the hand were cut (Fig. $2 A-C$ ). The reconstructed transverse view of the spinal cord after both lesions indicated that a lateral portion of the cuneate fasciculus was spared (Fig. 2C). Consistent with this observation, CTBlabeled foci from three digits (shown as three patches) on the lesioned side (right side) could be identified in expected locations on a few brainstem sections (Fig. $2 D$ ), but the density of the label was greatly reduced. In contrast, labeled foci from the three digits in the normal cuneate nuclei (left side) were very strong and topographically organized (dark patches). We concluded that in this animal, the lesions greatly reduced the quantity of inputs from the hand, but sparse inputs from digits 1, 3, and 5, and possibly other parts of the hand, remained.

$S M-D$

Afferents to the cuneate fasciculus were less extensively sectioned in SM-D, where a lesion was placed at the C5-C6 junction (Fig. $3 A, B)$. The lesion was placed caudal to most of the inputs from digit 1 , as the labeled inputs from digit 1 in the dorsal horn of the spinal cord were clearly rostral to the lesion (Fig. $3 B$ ). As the lesion was in the middle of the expected dorsal horn territory of inputs from digit 2, some inputs from digit 2 were likely spared. Two levels of horizontal sections through the spinal cord are included (Fig. 3B) to show the lesion at a more superficial level (Fig. 3B, left, section 17) and a deeper level (Fig. 3B, right, section 23). The reconstruction of the lesion, based on a series of sections, showed that the lesion was fairly complete, except for a superficial region of the cuneate fasciculus and much of the more medial gracile fasciculus. The lesion extended well into the central gray of the spinal cord without resulting in obvious behavioral consequences in addition to those expected from the location of dorsal column damage. Injections of CTB into the distal phalanges of digits 1,3 , and 5 resulted in densely labeled foci in the expected locations in the cuneate nucleus of the intact side. However, labeled foci corresponding to digits 3 and 5 were less densely labeled, and the level of label for digit 1 was reduced. We conclude that the lesion in SM-D had limited impact on the inputs from digit 1 , and only moderately reduced the inputs from digits 3 and 5 and possibly other parts of the hand.

\section{Quantification of labeled inputs to the cuneate nucleus and the correlation to cortical responsiveness}

To evaluate the effectiveness of the dorsal column lesion in each animal, we further quantified the differences in CTB label transported to terminal axon arbors in the cuneate nucleus on both the lesioned and nonlesioned sides of the brainstem by determining the cross-sectional area of the labeled foci in individual brainstem sections from caudal to rostral through the nuclei (Fig. 4). The measured foci were those areas that exceeded a threshold of optical density measured with NIH ImageJ software (see Materials and Methods). These procedures allowed us to quantitatively compare estimates of the proportions of sparse dorsal column afferents for three cases. While these estimates are based on afferents from digits 1,3 , and 5 only, they likely apply to all dorsal column afferents from the hand, and the results of recordings from cortex do not suggest that the lesions selectively avoided afferents from digits 2 and 4 and the palm.

SM-O had a nearly complete lesion of the dorsal columns when serial sections through the cuneate nuclei were examined. Sections through the length of the cuneate nucleus had foci of the label from the three injected fingers on the intact side, but only two sections had measurable labels on the lesion side (Fig. $4 \mathrm{~A}$, blue bars). Across sections, the areal extent of the measurable label on the lesioned side was $<1 \%$ of that intact side, and this difference was highly significant $(p<0.0001$, Wilcoxon matchedpairs signed-rank test). Moreover, the density of the few labeled axon patches was much less on the lesion side than the matching patches on the normal side (Fig. 1). Thus, the $1 \%$ value overestimated the amount of the remaining measurable first-order axons. 
CTB-labeled area of digits 1,3 , and 5 in the $\mathrm{CuN}$ of the brainstem

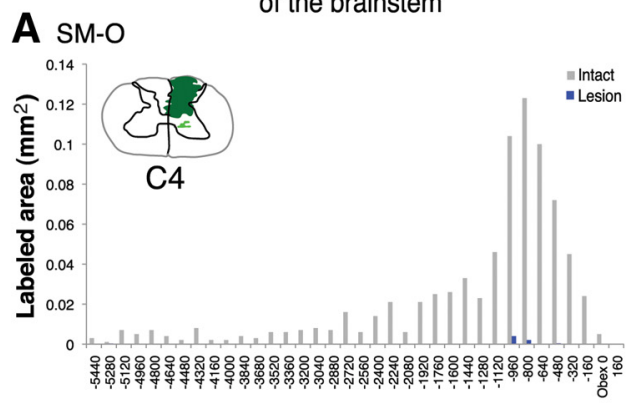

a

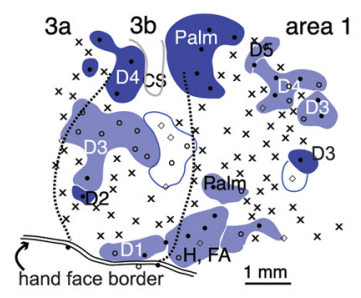

B SM-C

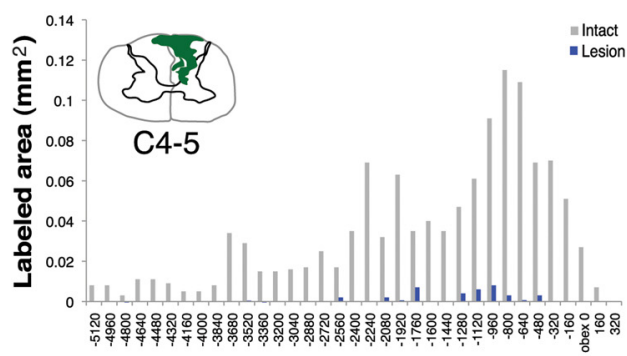

b

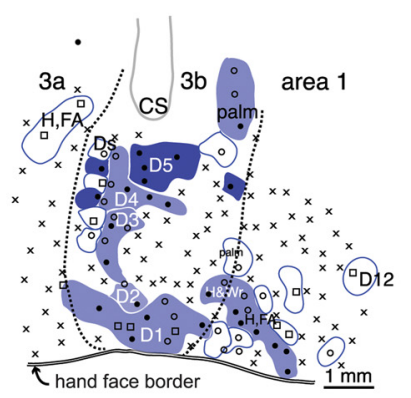

c
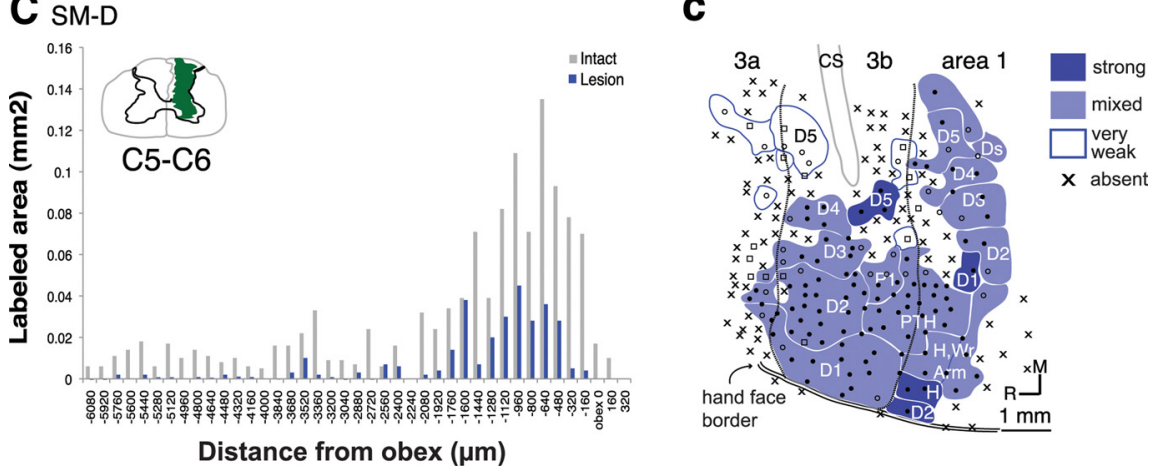

Figure 4. $\quad A-C$, Bar graphs showing the areal size of (TB-labeled axon arbor foci in the cuneate nuclei of the brainstem from three lesioned monkeys. The numbers on the $x$-axis are the distance (in micrometers) measured from the beginning of the obex. The negative values indicate the measured distances were caudal to the obex. The values on the $y$-axis are the areal size (in square millimeters) of the combined foci of (TB label for each section through the cuneate nucleus of the brainstem. Values from the intact and lesioned sides are compared. $\boldsymbol{a}-\boldsymbol{c}$, Topographical maps of hand territories in areas $3 \mathrm{~b}$ and 1 of the same three monkeys in $\boldsymbol{A}-\boldsymbol{C}$ depict regions where neurons had weak to strong responses to tactile stimulation. The $\mathrm{x}$ marks penetrations with unresponsive neurons. Neuronal response sites for different parts of the hand are grouped and outlined, and territories for D1 to D5 and palm pads are indicated. Shadings from dark to white indicate the proportion of good responses for each responding zone of the hand from high to low. 3a, Area 3a; 3b, area 3b; 1 , area 1; $C$, central sulcus; $F A$, forearm; $G R$, good response; $H$, hand; $M$, medial; $P 1$, pad 1 ; PTH, palm pad thenar; $R$, rostral; WR, weak response; Wr, wrist.

With $<1 \%$ spared hand inputs, a large number of electrode penetrations (Fig. $4 a$, black " $\mathrm{x}$ ") in areas $3 \mathrm{~b}$ and 1 recorded no responses to hand stimulation. However, within the hand representation regions of areas $3 \mathrm{~b}$ and 1 , some recording sites were activated by tactile stimuli. Although no label in the cuneate nucleus was attributed to the injection in the glabrous tip of digit 1 , three sites in area $3 \mathrm{~b}$ had neurons that responded well to touch on the dorsal surface of digit 1 . This suggests that the injection of tracer into the glabrous skin of digit 1 did not spread to involve the dorsal skin. Other regions of area $3 \mathrm{~b}$ responded well to digits 2 and 4, and weakly to digit 3, while none were activated by stimulating digit 5 . Similarly in area 1 , a small number of penetration sites responded to tactile stimulation of digits 3,4 , and 5 as well as palm or forearm. Given the completeness of the spinal cord lesion, the overall responsiveness of the hand regions in both areas $3 \mathrm{~b}$ and 1 to inputs from the hand was greater than expected.
However, compared with other animals with less extensive lesions (Fig. $4 B, b, C, c$ ), the proportion of recording sites that were responsive to the hand was much smaller.

SM-C appeared to have some sparing of axons in the cuneate fasciculus. The labeling of the cuneate nucleus after injections in digits 1,3 , and 5 was very sparse (Fig. 4B, scattered blue bars) compared with the intact side (Fig. $4 B$, gray bars), but was more than that of the lesion side in case SM-O (Fig. 4A). Sparsely labeled locations in the cuneate nucleus likely reflected inputs from digits 1,3 , and 5 , but the overall extent of the measured patches of labeling on the lesion side was only $2.2 \%$ of that on the intact side. The difference of labeled terminal areas in the cuneate nuclei between the two sides was highly significant $(p<0.0001$, Wilcoxon matchedpairs signed-rank test). The density of the labeled patches was also reduced on the lesion side compared with that on the normal side (Fig. 2), suggesting that even fewer of the afferents from digits 1,3 , and 5 remained. Contralateral to the lesion, large extents of both areas $3 \mathrm{~b}$ and 1 were unresponsive to tactile stimuli (Fig. $4 b$, black " $\mathrm{x}$ "). Compared with SM-O, however, more penetration sites were responsive to tactile stimulation of digits $1-5$ (Fig. 4b), suggesting that the sparse patches of labeling from these digits in the contralateral cuneate nucleus were effective in reactivating neurons in area $3 b$. Another region of area $3 \mathrm{~b}$ responded to digit 4 , suggesting that some digit 4 inputs were preserved as well. Surprisingly, in area 1, digit stimulation evoked only weak responses in a few penetration sites, and the strongest responses were limited to those evoked by stimulating the wrist and forearm.

SM-D had the most incomplete lesion of the cuneate fasciculus due to a section at a slightly lower level (C5-C6, between digits 1 and 2) in the dorsal column of the spinal cord. Foci of the CTB label from digits 1, 3, and 5 in the cuneate nuclei were found in many sections on the lesioned side, although the labeled patches were smaller and less widely distributed across sections than on the intact side (Fig. 4C). Quantitative measurement of the sizes of labeled axon terminal fields in the cuneate nucleus of the brainstem revealed a significant reduction ( $p<0.0001$, Wilcoxon matched-pairs signed-rank test) on the lesion side. Overall, the areal extent of labeled patches from digits 1,3 , and 5 on the lesion side was $23.4 \%$ of that on the intact side. Since most of peripheral afferents from D1 that entered the right side of the spinal cord were above the lesion, they were largely spared. As the labeled patches from the D1 injection were distinguishable from those from the D3 and D5 injections in several brainstem sections (Fig. 3D), we measured the sizes and densities of labeled patches of axon terminals for D1 territories in the cuneate nuclei on both sides of the brainstem. Both the total size 


\section{A CTB-labeled area of D1 territory in the CuN of the brainstem}

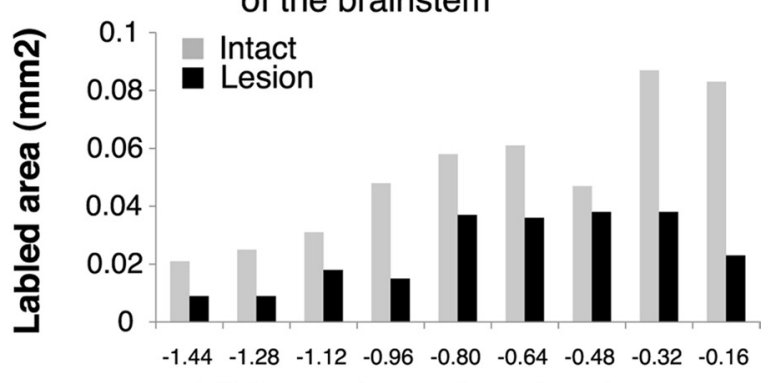

Distance from obex $(\mathrm{mm})$

\section{B CTB-labeled density of D1 territory in the} $\mathrm{CuN}$ of the brainstem

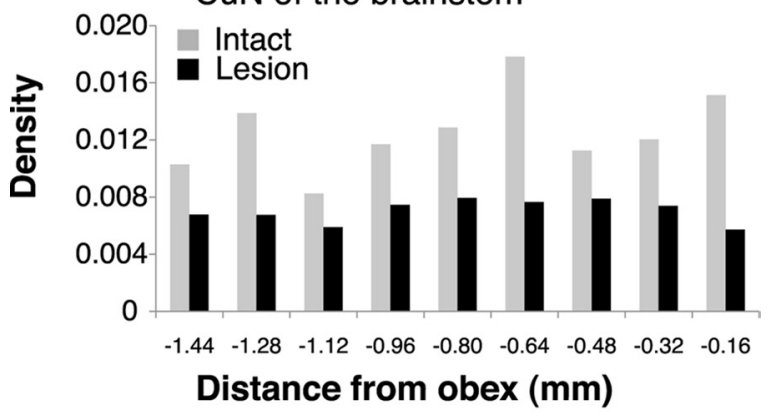

\section{CTB-labeled area in the dorsal horn of the spinal cord} สู

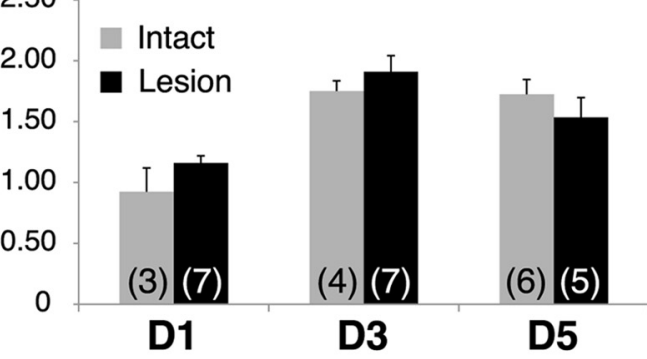

D CTB-labled density in the dorsal horn of the

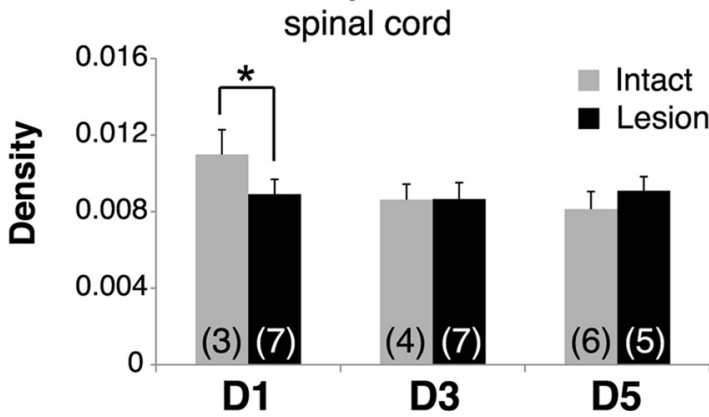

Figure 5. $A-D$, Bar graphs showing the relative extents and densities of CTB-labeled axon arbors in the digit 1 territory of rotunda subdivision of the cuneate nuclei $(\boldsymbol{A}, \boldsymbol{B})$, and the dorsal horn of the spinal cord $(\boldsymbol{C}, \boldsymbol{D})$ of SM-D. The value of label density was defined by inversed lightness of averaged values of reflected light from red, green, and blue channels (see Materials and Methods). The Wilcoxon matched-pairs signed-rank test was used to compare the difference between lesioned side with the intact side. $p<0.05$ is considered significant. $A, A$ comparison of the areal extent of the foci of label from D1 in the cuneate nucleus of the lesioned and intact sides. Measurements were taken from the middle-rostral sector of the cuneate nucleus where the rotunda subdivision is located as this is where CTB-labeled foci from digits 1,3 , and 5 could be distinguished on both sides. $\boldsymbol{B}$, A comparison of the densities of the foci of label for digit 1 in the rotunda subdivision of the cuneate nucleus. $C$, The areal extent of foci of labels from D1, D3, and D5 in the dorsal horn of the spinal cord of the two sides. There are no significant and density of labeled patches for D1 were significantly reduced $(p<0.004$ for both measurements) on the lesion side (areal size: $0.023 \pm 0.0126 \mathrm{~mm}^{2}$; density: $\left.0.007 \pm 0.0008\right)$ compared with the intact side (areal size: $0.048 \pm 0.0236 \mathrm{~mm}^{2}$; density: $0.012 \pm$ $0.003)$. Based on this calculation, the first-order afferents from digit 1 were reduced by about half (Fig. $5 A, B$ ). For comparison, similar measurements were made from patches of label in horizontally cut spinal cord sections. The values were used to compare the sizes and densities of CTB-labeled patches for digit territories 1, 3, and 5 in the dorsal horn of the spinal cord. The results indicate that the sizes and the densities of D3 and D5 territories were similar on both sides on the dorsal horn. However, as shown in Figure 5, $C$ and $D$, the labeled axon territory of D1 in the dorsal horn was slightly larger and density was significantly lower on the lesion side than on the normal side $(p<0.04)$. These results suggested that some of the peripheral afferent inputs from D1 were interrupted by the lesion at cervical segments C5-C6, even though the section was below the main focus of D1 input in spinal cord.

Recording from somatosensory cortex in this monkey revealed some unresponsive zones, but also revealed large regions of hand cortex in both areas $3 \mathrm{~b}$ and 1 that were responsive to touch on each of the five digits (Fig. 4C). Even though $>75 \%$ of the activating inputs from the hand were lost, the remaining inputs were highly effective in reactivating neurons in areas $3 \mathrm{~b}$ and 1.

In summary, across these three animals, less cortical reactivation followed the larger lesions of the cuneate fasciculus of the spinal cord.

The somatotopic organizations of areas $3 \mathrm{~b}$ and $1 \mathrm{hand}$ regions contralateral and ipsilateral to dorsal column lesions

To characterize the changes in digit somatotopy after contralateral dorsal column lesions, we compared the neuronal responsiveness and RF properties of neurons between the input-deprived contralateral area $3 \mathrm{~b}$ and nondeprived ipsilateral area $3 \mathrm{~b}$ in the same monkeys. Mapping the ipsilateral hand region also served as a control to assure that the weak responses encountered in the deprived cortex were not due to longer hours of recording under isoflurane anesthesia. The recordings from the deprived cortex preceded the recordings from the intact sides. As an independent control, we also mapped the hand representations of areas $3 \mathrm{~b}$ and 1 in one surgical control monkey who received a lateral spinal cord lesion that did not affect the dorsal columns. Control maps obtained from four hemispheres are presented in Figure 6.

Topography and neuronal receptive field properties in nondeprived cortex

Similar to previous findings (Sur et al., 1982), we observed topographically organized maps of digit representation maps and strong responses to tactile stimulation in nondeprived cortex in all four hemispheres (Fig. 6). Somatotopically, the tips of digits

differences in the extents of labeling in the spinal cord on the two sides, indicating that the dorsal column lesion did not disrupt most of the terminations in the dorsal horn. $\boldsymbol{D}$, However, a comparison of the densities of the labeled foci in the dorsal horn of the spinal cord after injections in digits 1,3 , and 5 reveal that only the foci for digit 1 were significantly less dense on the lesioned side compared with the intact side, suggesting the lesion at the C 6 level (Fig. 3) may have disrupted some of the D1 afferent terminations in the dorsal horn. ${ }^{*} p<0.05$. No significant differences were observed for foci for digits 3 and 5 . Values in $\boldsymbol{C}$ and $\boldsymbol{D}$ are reported as the mean $\pm S D$. 

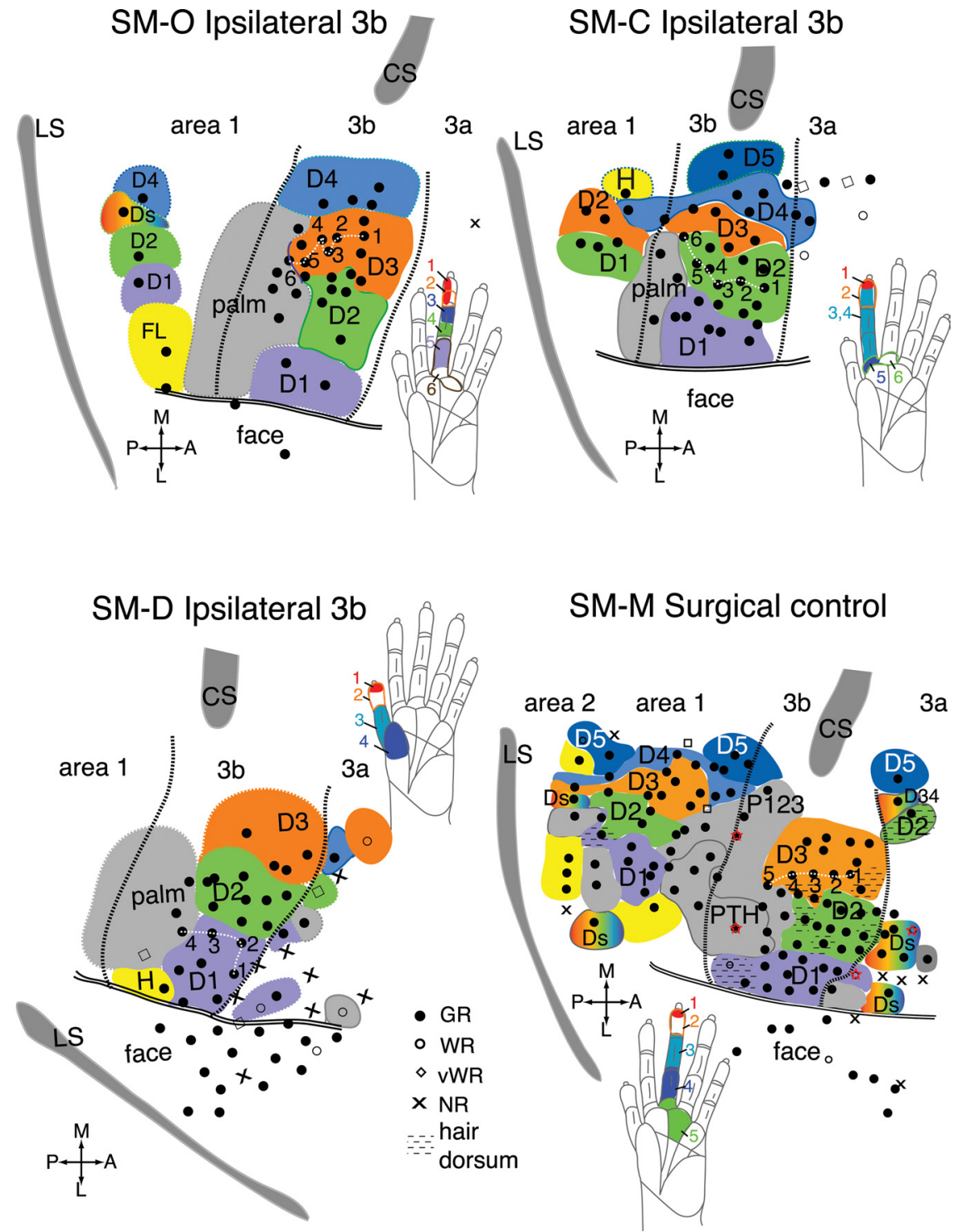

Figure 6. The organization of digit representations of area $3 \mathrm{~b}$ in three squirrel monkeys on the side ipsilateral to lesions of the dorsal columns of the spinal cord. The fourth squirrel monkey (SM-M) was a surgical control monkey in which a laminectomy was performed at the (4-C5 level of cervical vertebrae; however, the dorsal columns were not touched. Maps of each case were reconstructed based on the locations of receptive fields for neurons at each electrode penetration (see Materials and Methods). The schematic drawings of hands for each case depict the examples of the neuronal receptive fields as they progressively shifted from distal to proximal portions of the digits as electrode penetrations moved from rostral to caudal in cortical area $3 \mathrm{~b}$. The solid dots mark good responses; the open circles mark weak responses; the open diamonds mark very weak responses to hard taps; the dashed regions mark responses to hairy or dorsal skin; x marks microelectrode penetrations with no responses; stars mark electrolytic lesions. In all cases, the vast majority of responses in areas $3 \mathrm{~b}$ and 1 were good, and the somatotopic organizations of areas $3 \mathrm{~b}$ and 1 appeared to be normal. A, Anterior; M, medial; L, lateral; P, posterior. For other conventions, see Figures 1 and 4.

1-5 were represented in a lateromedial sequence from D1 to D5 along the rostral border of area $3 \mathrm{~b}$ and the caudal border of area 1 , with more proximal phalanges of the digits and pads of the palm in between (for examples, see Fig. 6). Only an occasional recording site was found where neurons responded to the dorsal hairy surface of the hand and digits. Overall, the two hand representations approximately constituted mirror images of each other in somatotopy, reversing at the area $3 \mathrm{~b} / 1$ border (Fig. $6 D$ ). It was quite clear that almost $100 \%$ of the mapping sites in the digit region of area $3 \mathrm{~b}$ produced good or very good responses (Fig. 6, solid dots). The depths at which strong responses were first encountered within the electrode penetrations were usually between 500 and $700 \mu \mathrm{m}$ below the pial surface. Based on these findings, we believe that the weak responses or lack of responses in the deprived hand region of areas $3 \mathrm{~b}$ and 1 were due to the interruption of peripheral inputs in the dorsal column of the spinal cord, and not artifacts of long periods of anesthesia.

Topography and neuronal receptive field properties in deprived cortex

After 7-9 weeks of postlesion survival time following effective sectioning of the afferents from the hand in the dorsal columns of the spinal cord, the deprived somatosensory areas $3 \mathrm{~b}$ and 1 were mapped under isoflurane anesthesia. In general, the deprived cortex was partly responsive but not fully reactivated, topography was abnormal, the neuronal responses were sometimes weak, and receptive fields were sometimes discontinuous and relatively large. In addition, the depths of the most responsive recording sites tended to be deeper (usually $\sim 700-1000 \mu \mathrm{m}$ below pia surface, and likely within layer 4) than the first strongly responsive sites on the normal side (usually 500-700 $\mu \mathrm{m}$, and likely within layer 3 and the beginning of layer 4). This estimate of depth in relation to architectural cortical layers is consistent with the laminar activity profile study of area $3 \mathrm{~b}$ in squirrel monkeys by Schroeder et al. (1995). The tendency to find strong responses at more superficial recording depths in the normal compared with the deprived hemispheres reflects the stronger activations of normal hemispheres.

\section{$S M-O$}

Hand regions in areas $3 \mathrm{~b}$ and 1 of both hemispheres in this monkey were mapped 7 weeks after an extensive unilateral dorsal column lesion at cervical level 4. Peripheral afferents from all digits entered below the lesion, and $<1 \%$ of the primary afferents survived (Fig. 4A). The hand representations in deprived areas $3 \mathrm{~b}$ and 1 were abnormal in that neurons in most penetrations were unresponsive to somatosensory stimuli (Fig. 7). Only three sites had neurons responsive to digit 1 , and the receptive fields were on the dorsal surface of the digit, which is normally poorly represented. Only one site responded to digit 2, while several sites had neurons with receptive fields on digit 3 , and a few had neurons that responded to digit 4 . The receptive fields for these sites were not unusually large, and they approximately reflected the somatotopy of a normal representation in areas $3 \mathrm{~b}$ and 1 . However, unresponsive zones in area $3 \mathrm{~b}$ were in the locations expected for some representations of the skin surface including the glabrous skin of digit 1, most of digit 2, and some parts of glabrous digits $3-5$. Sites in area 1 were activated during digit 5 stimulation, but corresponding sites for digit 5 stimulation in area $3 \mathrm{~b}$ were not 
found. In contrast to the weak responses encountered in the deprived hand representations of areas $3 \mathrm{~b}$ and 1 , all the mapping sites (marked with dots) in the more lateral face representations responded very well to light tactile stimuli on the face (Fig. 7).

\section{$S M-C$}

Hand regions in areas $3 \mathrm{~b}$ and 1 of both hemispheres in this monkey were mapped 9 weeks after a second, more effective unilateral dorsal column lesion of the spinal cord. We estimated that the two lesions together removed $98 \%$ of the terminations of first-order afferents from the hand in the cuneate fasciculus (Fig. 4B). Nevertheless, the five digits were represented in a normal lateromedial sequence of D1-D5 along the rostral border of area 3b (Fig. 8). Many of the responses were weak, and much of the area $3 \mathrm{~b}$ hand representation was unresponsive. Higher than expected proportions of receptive fields were found on the dorsal surfaces of digits. Most parts of the glabrous skin were not represented in the deprived area $3 \mathrm{~b}$, and there were only a few penetrations where RFs on either the tip or distal phalanges of glabrous digits could be found (Fig. 8). Even fewer sites were activated in area 1, where lateral recording sites responded to the forearm and hand or, weakly, to digit 1. In contrast to area $3 b$, none of the receptive fields for neurons in area 1 were on the glabrous surface of digits 2-5.

\section{$S M-D$}

The deprived cortical areas 3b, 1, and 3a, and the normally activated area $3 \mathrm{~b}$ of this monkey were mapped 8 weeks after a unilateral section of the right cuneate fasciculus. The lesion was at a slightly lower spinal cord level (C5-C6) than the other two cases. This level allowed most of the inputs from digit 1 to enter the cuneate fasciculus above the lesion, as well as some of the inputs from digit 2 and perhaps even digit 3 . Transported tracer from digits 1,3 , and 5 on the lesion side revealed that inputs to the cuneate nucleus from digit 1 were somewhat reduced, and only some inputs from digits 3 , possibly 4 , and 5 remained (Fig. 3). Thus, the lesion was extensive, and we estimate that $<25 \%$ of the afferents from the hand in the cuneate fasciculus remained (Fig. 4C). As a result of an extensive but incomplete loss of afferents, the lateral parts of the hand representations in area $3 \mathrm{~b}$ that normally respond to digits 1 and 2 and the thenar pad of the palm continued to respond well to touch on those parts of the hand (Fig. 9). The more deprived medial parts of the hand territories of areas $3 \mathrm{~b}$ and 1 had
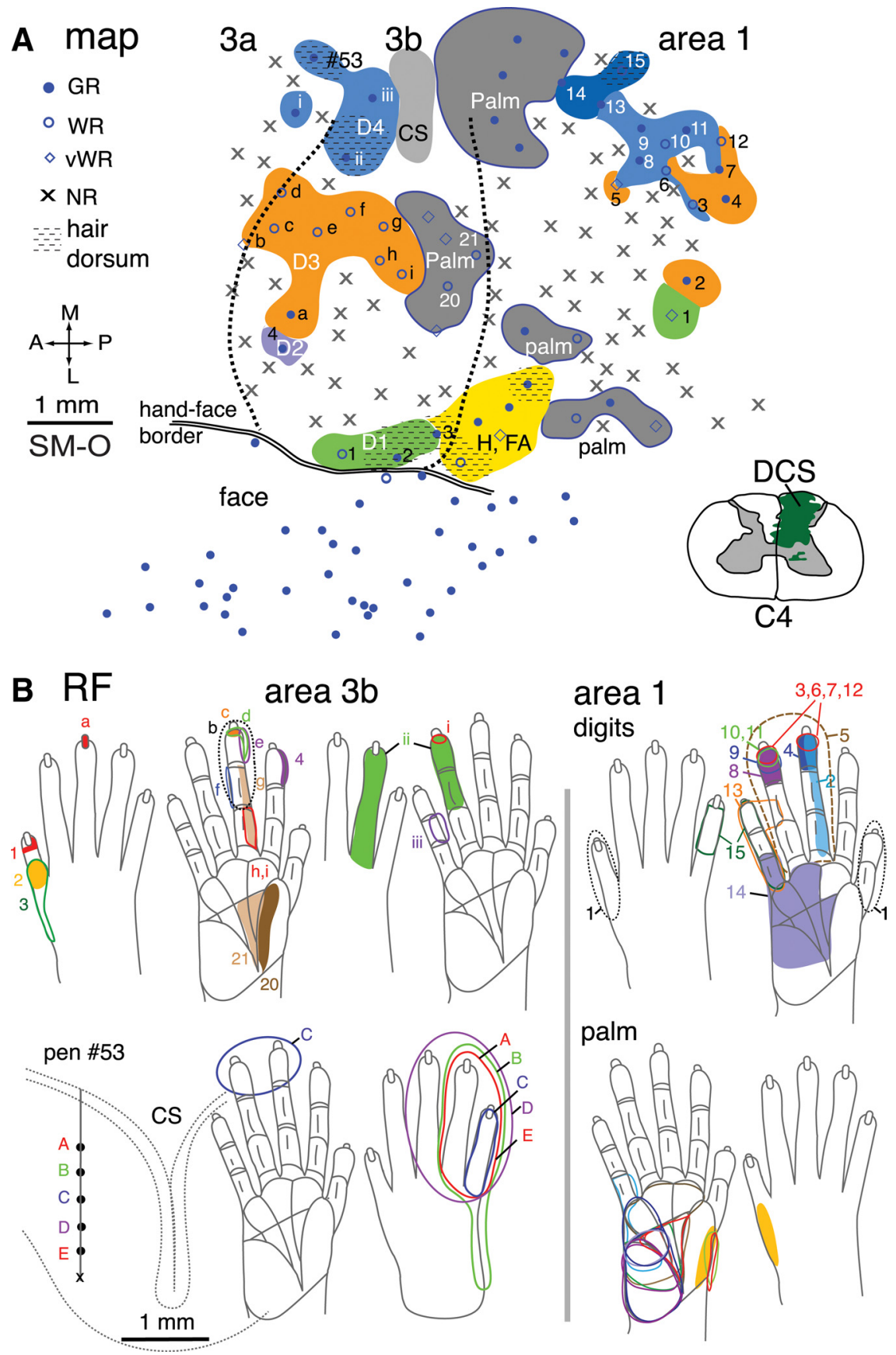

Figure 7. The somatotopic map of areas $3 \mathrm{~b}$ and 1 of a squirrel monkey (SM-0) obtained 7 weeks after section of the contralateral dorsal columns. $A$, The somatotopic map shows the locations of microelectrode penetrations. Solid dots mark penetrations with good responses; black open circles mark those with weak responses; black diamonds mark those with very weak responses to hard taps; $x$ marks microelectrode penetrations with no responses. Heavy black dashed lines mark area boundaries based on myelin architecture. Note that the vast majority of microelectrode penetrations over unaffected face cortex are marked with good responses. $\boldsymbol{B}$, Receptive fields obtained with microelectrode recordings are outlined on the drawing of the glabrous and dorsal sides of the hand of SM- 0 . The dashed lines on the drawing of hand indicate that the exact boundaries of the RFs were hard to define due to very weak responses to hard taps. Numbers or letters on the drawings of the hands indicate the receptive fields recorded from matching microelectrode penetrations in the map. Areas 3a, 3b, and 1 are indicated. CS, Central sulcus; dD1 and dD2, distal phalange of $D 1$ and $D 2$, respectively; $G R$, good response; $M$, medial; $N R$, no response; $P D 4$, proximal phalange of $D 4 ; R$, rostral; $P 2$, pad 2; PTH, pad thenar; WR, weak response; vWR, very weak response to hard taps. For other conventions, see Figures 4 and 6. regions of unresponsive neurons (Fig. 4c). Nevertheless, all five digits were represented in lateromedial sequence from D1 to $\mathrm{D} 5$ on both areas $3 \mathrm{~b}$ and 1 , as in normal monkeys. However, there were a number of abnormal features in the somatotopic patterns. 
The representations of digits 1 and 2 in area $3 \mathrm{~b}$ were unusually large, suggesting that they had expanded into deprived regions of cortex (Fig. 9). While the representations of digits 1 and 2 in area $3 b$ were quite large, the representations of digits 1 and 2 in area 1 were surprisingly small, and they were displaced medially from their normal, more lateral locations (Fig. 10). There were only three penetrations in area 1 where neurons responded to light touch on digit 1 , and only three penetrations for digit 2. The large representation of the hand, wrist, and arm in the lateral part of area 1 was also an unusual feature. Inputs from the thenar pad and palm pad 1 were probably partly spared by the C5-C6 lesion, and they had expanded territories in areas $3 \mathrm{~b}$ and 1 (Figs. 9, 10). The receptive fields of neurons in the deprived regions of digits 3-5 were relatively large, and usually included multiple digits or phalanges (Fig. 9). In contrast, the receptive fields of neurons in the less deprived regions of digit 1 and part of digit 2 were small.

Finally, the rostrocaudal progressions of somatotopy in the highly responsive D1 and D2 territories in area $3 \mathrm{~b}$ were abnormal. To illustrate this, we subdivided the D1 and D2 territories of area $3 \mathrm{~b}$ into rostral, middle, and caudal zones on digit 1 (D1r, D1m, D1c, respectively) and digit 2 (D2r, D2m, D2c, respectively). Here, we found that the representations of the distal parts of these digits were not limited to the rostral part of area $3 \mathrm{~b}$ as reported in early studies of normal monkeys (Sur et al., 1982, Jain et al., 1997) and as also found in our control and normal hemispheres (Fig. 6). Instead, neurons responsive to the distal portion of the digits were also found in the caudal portions of area $3 \mathrm{~b}$, where receptive fields on digit tips intermingled with those on the proximal part of digits. These results indicated that even though the inputs from digits 1 and 2 were largely spared, their representations in the deprived area $3 \mathrm{~b}$ were not normal.

\section{Comparison of digit somatotopy in area} $3 \mathrm{~b}$ and area 1

In normal monkeys, areas $3 \mathrm{~b}$ and 1 share many common features in topography, preferred stimulus, and RF properties. However, we found that the patterns of somatotopy and the extents of reactivation were different between areas $3 \mathrm{~b}$ and 1 after dorsal column lesions. Thus, there were larger unresponsive zones in area 1 compared with area 3b (Figs. 7, 8). Receptive fields for neurons in area 1 were usually larger and more often involved multiple

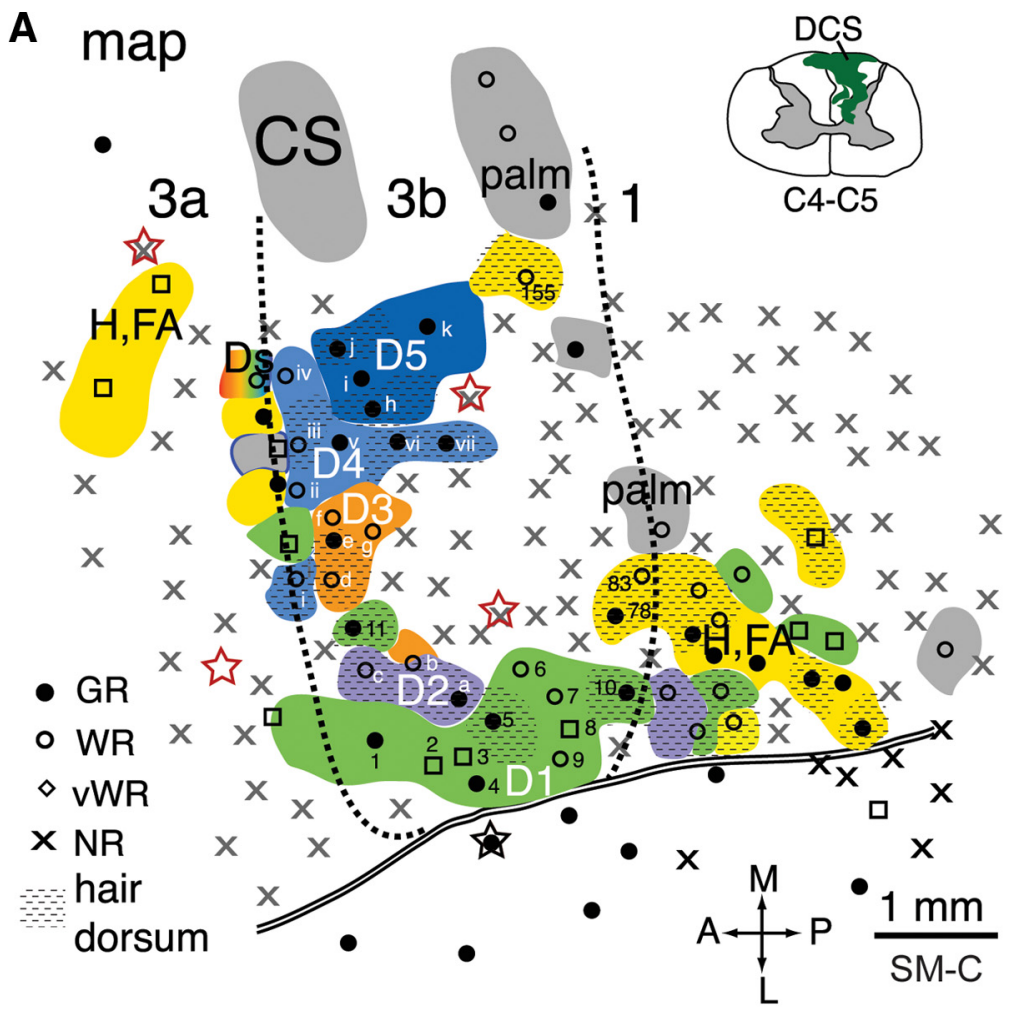

\section{B \\ RF \\ area $3 b$}

D1

D2,D3
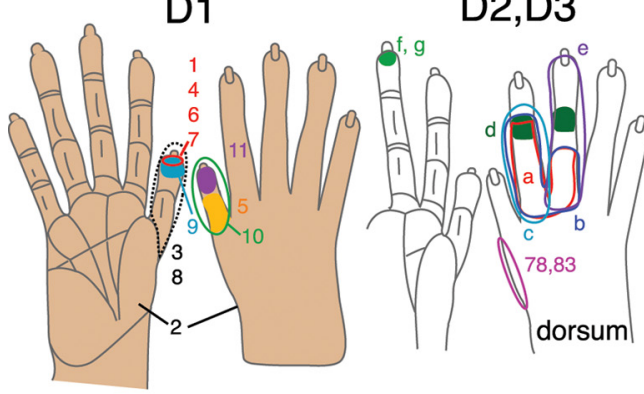

D4
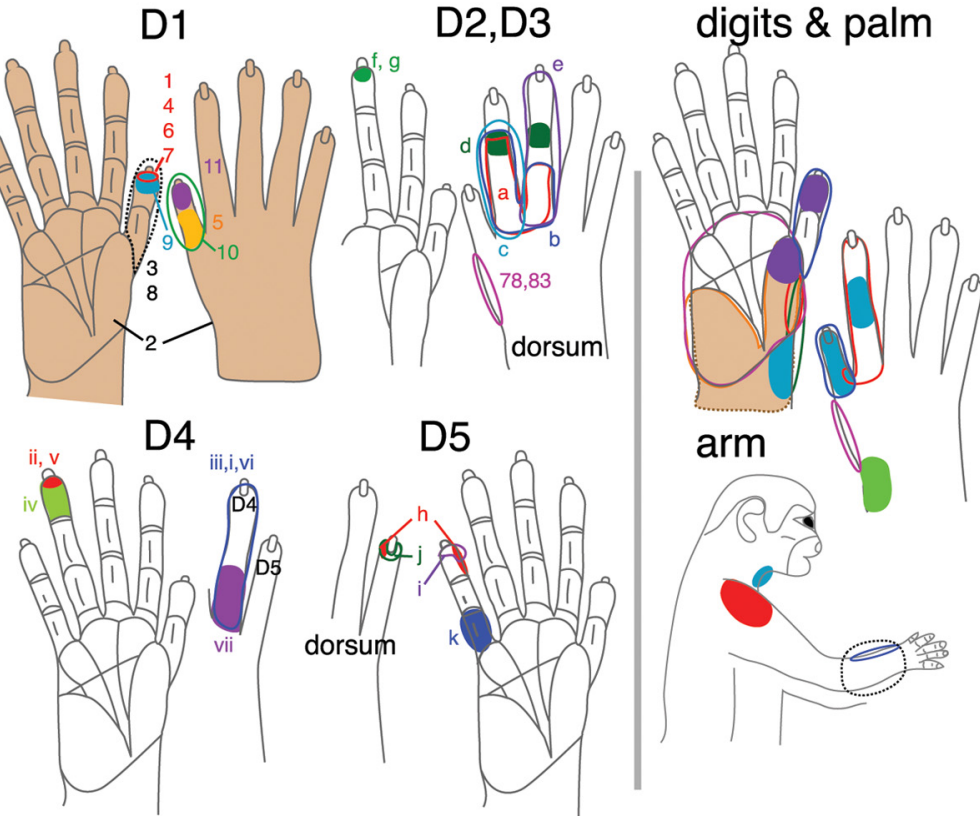

Figure 8. The somatotopic map obtained 9 weeks after a second, effective lesion of the contralateral dorsal columns in a squirrel monkey (SM-C). $\boldsymbol{A}$, The cortical map of somatosensory areas $3 \mathrm{~b}$ and 1 shows the locations of microelectrode penetrations, responsiveness, and nonresponsive zones. Solid dots mark penetrations with good responses; black open circles mark those with weak responses; black diamonds mark those with very weak responses to hard taps; $\mathrm{x}$ marks microelectrode penetrations with no responses; heavy black dashed lines mark area boundaries based on myelin architecture. $\boldsymbol{B}$, Receptive fields obtained with microelectrode recordings are outlined on drawings of the glabrous and dorsal sides of the hand of a squirrel monkey. The dashed lines on the drawing of the hand indicate that the exact boundaries of the RFs were hard to define due to very weak responses to hard taps. Numbers or letters on the drawings of the hands indicate the receptive fields recorded from matching microelectrode penetrations in the map. For other conventions, see Figures 4 and 6. 
D1
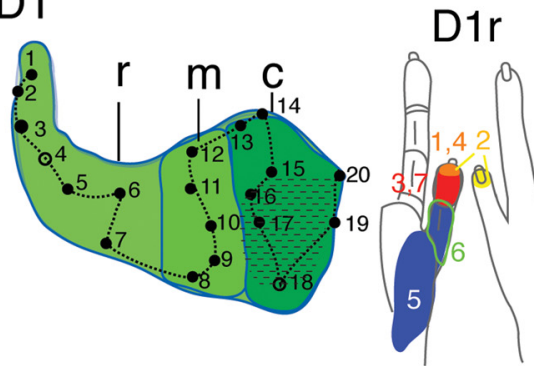

D2
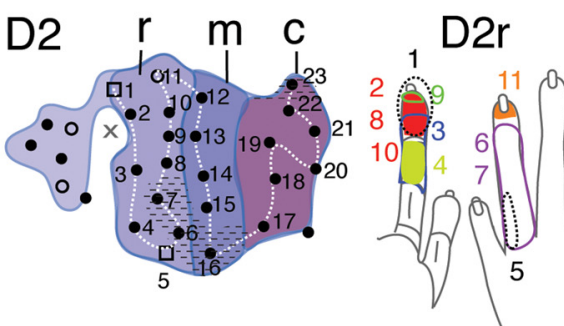

Mostly spared D1, D2
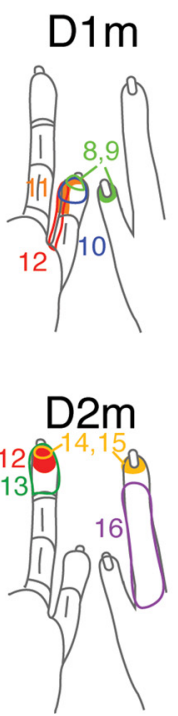

Area 3b RF
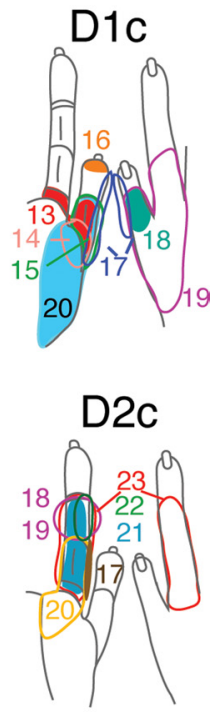

D3
Partially deprived D3-D5 • GR

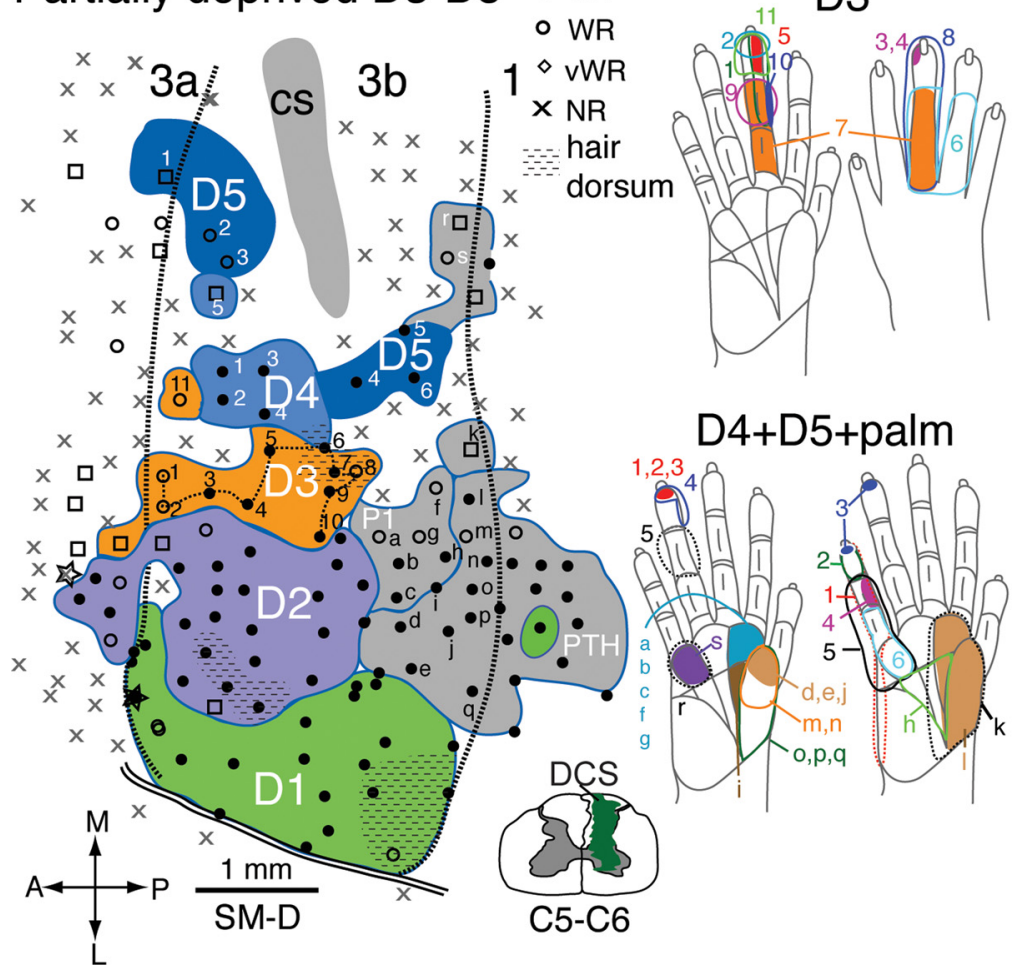

Figure 9. A somatotopic map obtained 8 weeks after a lesion of the contralateral dorsal columns in SM-D. On the left, solid dots mark penetrations with good responses; black open circles mark those with weak responses; black diamonds mark those with very weak responses to hard taps; $x$ marks microelectrode penetrations with no responses. On the right, receptive fields obtained with microelectrode recordings are outlined on drawings of the glabrous and dorsal side of the hand of a squirrel monkey. Numbers or letters on the drawings of the hands indicate the receptive fields matching microelectrode penetrations in the map. The detailed topographical organizations of the most spared digits 1 and 2 are shown on the left top two panels. By dividing the regions representing digits 1 and 2 into three sectors (rostral, middle, and caudal), we show that the representations of the distal part of digits are not limited to the rostral portions of digit representations of area 3b, as reported in normal monkeys (Sur et al., 1982), but they also appear in the middle and caudal sectors of area $3 \mathrm{~b}$. In the bottom half of the illustration, digits 3-5 were represented in partly deprived cortex in an abnormal fashion, reflected by the appearance of silent zones and abnormal somatotopy. The receptive fields did not progressively shift as the electrode penetrations moved systematically from rostral to caudal (e.g., D3 representation). In addition, the representations of D3-D5 were fragmented. For conventions, see Figures 4 and 6. phalanges (Figs. 7, 8, 10). While the representations of digits 1 and 2 were overrepresented in area $3 b$ in SM-D, their representations in area 1 remained quite small with only a few penetrations where digit 1 and 2 could be found in area 1 . Such results suggest that somatotopic reorganization of area 1 after peripheral sensory loss is often different and likely more variable than that of area 3b. Previously, area 1 reorganization after median nerve section in monkeys was described as different and more variable than area $3 \mathrm{~b}$ reorganization (Merzenich et al., 1983a,b).

In summary, the somatosensory areas $3 \mathrm{~b}$ and 1 underwent reactivation and reorganization after sectioning the contralateral dorsal columns at a high cervical level. With survival times up to 9 weeks after dorsal column lesions, deprived cortex was partly responsive but not totally reactivated, topography was not normal, the neuronal responses were sometimes weak, and the receptive fields of neurons were sometimes discontinuous and relatively large. The degree of reactivation corresponded to the amount of remaining dorsal column afferents that terminate in the cuneate nucleus of the brainstem. Considerable reactivation occurred even when $<1 \%$ of the primary afferent inputs remained in the cuneate nucleus of the brainstem. We did not encounter face representations in the hand region of area 3b. A possible explanation for this finding is that 7-9 weeks of recovery time after an effective dorsal column lesion may not be long enough to produce expansion of face representations in the deprived hand region in primary somatosensory cortex (Jain et al., 1997).

\section{Discussion}

We characterized the neuronal responsiveness and somatotopy of the hand representations in areas $3 \mathrm{~b}$ and 1 of somatosensory cortex of squirrel monkeys weeks after sectioning of the dorsal columns of the contralateral spinal cord. Such lesions immediately abolish the responsiveness of the hand representation to touch on the hand in areas $3 \mathrm{~b}$ and 1 of somatosensory cortex, but, over weeks of recovery time, responsiveness to touch on some parts of the hand returns to much of the deprived hand cortex, followed by responsiveness to touch on the lower face after even longer periods of 6-8 months (Jain et al., $1997,1998,2008)$. The return of such responsiveness has been considered to be due to the preservation of some dorsal column afferents from the hand, the growth of new connections in the dorsal 
A map
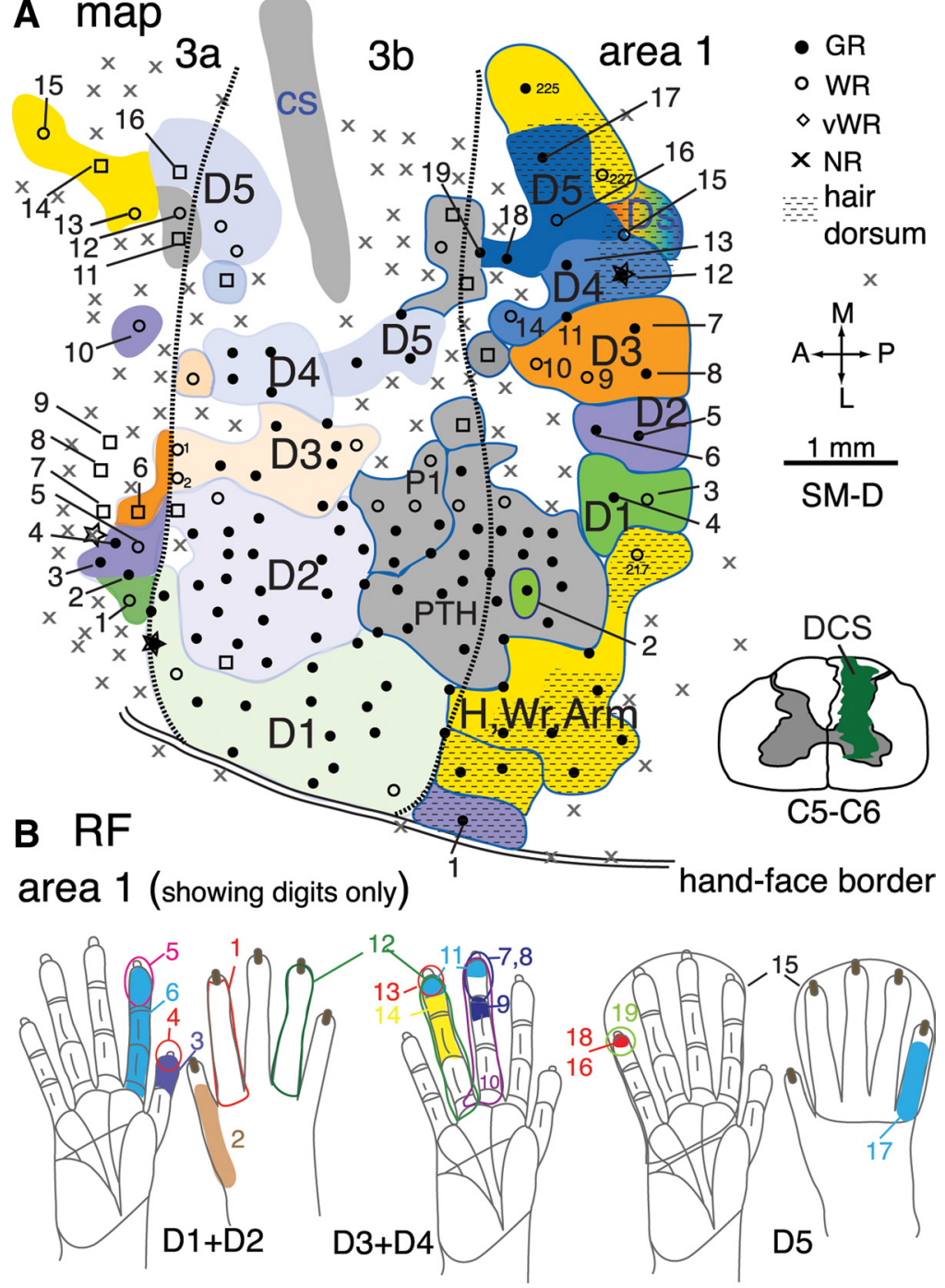

3a
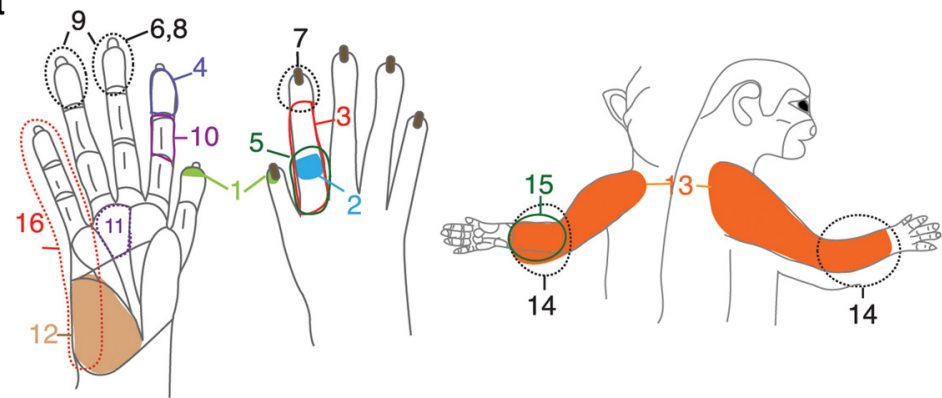

Figure 10. The somatosensory maps of areas $3 a$ and 1 and neuronal receptive fields on the forelimb in SM-D obtained 8 weeks after an incomplete lesion on the contralateral dorsal column. $\boldsymbol{A}$, The somatotopic map on the top shows the locations of microelectrode penetrations and electrolytic lesions (stars). Solid dots mark penetrations with good responses; black open circles mark those with weak responses; black diamonds mark those with very weak responses to hard taps; $x$ marks microelectrode penetrations with no responses; heavy black dashed lines mark area boundaries based on myelin architecture. $\boldsymbol{B}$, Receptive fields obtained with microelectrode recordings are outlined on drawings of the glabrous and dorsal side of the hand of a squirrel monkey. The dashed lines on the drawing of hand indicate that the exact boundaries of the RFs were hard to define due to very weak responses to hard taps. Numbers or letters on the drawings of the hands indicate the receptive fields recorded from the respective microelectrode penetrations in the map. For conventions, see Figures 4 and 6.

column nuclei; and the potentiation of those and other connections. Over longer periods of time, the growth of afferents from the face to the cuneate nucleus results in inputs from the face activating hand cortex and misperceptions of touch on the face being felt on the hand (Ramachandran et al., 1992; Ramachandran, 1993). Our present study adds to previous results by making the relationships between extents of cortical reactivation and dorsal column lesions clear. By using tracers subcutaneously injected into the skin of digits, we were able to study cortical activation several weeks after lesions disrupted 75 to $>99 \%$ of afferents from the hand in the dorsal columns. In each of three cases, some cortical reactivation occurred in area $3 \mathrm{~b}$, although the somatotopy was incomplete and abnormal. In addition, the reactivation in area 1 differed in extent and somatotopy from that in area $3 \mathrm{~b}$. The reactivations of somatosensory cortex in our experimental monkeys were confirmed by optical imaging and highresolution 9.4 T fMRI. In addition, hand use was impaired immediately after the larger spinal cord lesions, but greatly improved over weeks of postoperational testing. These additional imaging and behavioral results will be presented elsewhere.

\section{The effectiveness of our spinal cord lesions in eliminating first-order afferents from the hand}

In the present study, we were able to quantify the extents of preserved firstorder afferents by labeling afferents from the digits of the hand with CTB, and determining the locations and magnitudes of these terminations in the spinal cord and cuneate nucleus on the side of the spinal cord lesion in comparison with the intact side. Because afferents from specific skin locations terminate in specific locations in the dorsal horn of the spinal cord and in the dorsal column nuclei of primates with little if any overlap from adjacent skin locations (Florence et al., 1988, 1989, 1991, 1994; Strata et al., 2003; Qi and Kaas, 2006), we were able to measure the remaining axon terminal fields in the cuneate nucleus of the brainstem, and directly compare the extents and locations of axon inputs on the lesioned and intact sides. Such quantifications have not characterized previous studies of this type. With a nearly complete lesion with even $<1 \%$ of the afferents from the hand remaining in the dorsal columns, parts of the hand representation in primary somatosensory cortex were reactivated, and this likely contributed to the recovery of hand use. In another monkey with nearly $24 \%$ of remaining fibers, more than half of the recording sites in the hand region of area $3 \mathrm{~b}$ encountered neurons responsive to stimuli on the hand (Figs. $4 c, 9$ ), and the majority of the responses were to low-threshold cutaneous stimulation. 


\section{Possible mechanisms of cortical reorganization after sensory loss}

In the somatosensory system of monkeys, a nerve cut, digit amputation, or dorsal root or dorsal column section deactivates a part of the representation of the hand in area $3 \mathrm{~b}$ of somatosensory cortex so that neurons are no longer responsive to tactile or other peripheral stimuli. Over weeks or months of recovery, preserved cutaneous inputs from the hand either expand their cortical territory of activation or gradually become effective in activating cortical neurons after an initial complete loss of activation (Merzenich et al., 1983a,b; Pons et al., 1991; Florence et al., 1996, 1998; Jain et al., 1997, 1998, 2000, 2008; Darian-Smith and Brown, 2000). The recovery of responsiveness is paralleled by improvements in hand use (Darian-Smith and Ciferri, 2005). Thus, the reactivation of cortex appears to have important functional consequences. Although the exact mechanisms of long-term plasticity after dorsal column lesion are not fully understood (for discussion, see Jain et al., 2008; Graziano and Jones, 2009), such long-term plasticity appears to involve the creation of new connections and an increase in the effectiveness of existing connections (Kaas, 2001; Kaas et al., 2008).

In the monkeys with more complete dorsal column lesions (e.g., SM-O), it seems unlikely that all of the cortical reactivation depends on the few $(<1 \%)$ remaining first-order afferents. However, the dorsal column nuclei also receive nonprimary inputs from neurons in the ipsilateral dorsal horn of the spinal cord (Rustioni et al., 1979; Bennett et al., 1983). The present results raise the possibility that some of the second-order afferents that originate caudal to dorsal column lesions avoid the lesion and join the dorsal columns at a more rostral level. While the paths of these second-order afferents to the dorsal column nuclei have not been fully determined, the lesions of the dorsal columns in the present study likely interrupted second-order afferents as well as first-order afferents, but more second-order afferents may have been preserved. Electrophysiological studies suggest that the secondorder neurons that contribute to the dorsal columns are excited by both tactile and noxious stimuli or tactile stimuli alone (Bennett et al., 1983). Second-order afferents to the cuneate nucleus are thought to activate intrinsic inhibitory neurons and thereby restrain the receptive field sizes of the cuneate relay neurons (Dykes and Craig, 1998). Possibly some second-order afferents become capable of activating relay neurons during recoveries following dorsal column lesions. In addition, the spinothalamic pathway could contribute to the reactivation of areas $3 \mathrm{~b}$ and 1 , although the results of some studies argue against this possibility (Dreyer et al., 1974; Olausson et al., 2008).

\section{Reactivation and reorganization of area 1}

Parts of area 1 were also responsive to tactile stimulation of the hand after behavioral recovery from dorsal column lesions in the present experiments. While area 1 has been found to be responsive to somatosensory stimuli after dorsal column lesions (Jain et al., 2008), the distribution and somatotopy of this reactivated representation have not been fully described. In the present study, we directly compared the responsiveness of the hand territories in areas $3 \mathrm{~b}$ and 1 , weeks to months after a range of large to nearly complete dorsal column lesions. We found that fewer locations in area 1 were responsive to touch on the hand than locations in area $3 \mathrm{~b}$. In addition, the types of responses differed in that skin locations that were highly effective in activating a number of sites in area $3 \mathrm{~b}$ could be rather ineffective in area 1 , and a few skin locations activated area 1 sites better than area $3 \mathrm{~b}$ sites. Thus, the somatotopy in area 1 less effectively mirrored that of area $3 \mathrm{~b}$ in lesioned monkeys compared with normal monkeys. Similar results were reported after monkeys recovered from a sensory loss in the hand following median nerve section (Merzenich et al., 1983a,b). As area 3b projects to layer 4 of area 1 (Vogt and Pandya, 1978), inputs from area $3 \mathrm{~b}$ are thought to be the main source of activation in area 1 (Juliano et al., 1990), and lesions of area $3 \mathrm{~b}$ abolish evoked responses in area 1 (Garraghty et al., 1990). However, it remains possible that inputs to layer 3 of area 1 from the ventroposterior nucleus (Cusick et al., 1985) gain synaptic strength and become capable of activating area 1 during recoveries from dorsal column lesions in monkeys, so that activation patterns in area 1 become somewhat independent of those in area 3b. After long-standing but possibly incomplete lesions of the hand representation in area $3 \mathrm{~b}$ of macaque monkeys, Randolph and Semmes (1974) reported that area 1 responded to tactile stimuli.

Overall, the present results, together with previous findings, indicate that nearly complete lesions of dorsal column afferents do not permanently deactivate somatosensory cortex. Even a few surviving afferents can have a large role in reactivating cortex. Second-order afferents may also have an important role in reactivation and behavioral recoveries.

\section{References}

Almli CR, Finger S (1988) Toward a definition of recovery of function. In: Brain injury and recovery: theoretical and controversial issues (Finger S, Levere TE, Almli CR, Stein DG, eds), pp 1-14. New York: Plenum.

Bennett GJ, Seltzer Z, Lu GW, Nishikawa N, Dubner R (1983) The cells of origin of the dorsal column postsynaptic projection in the lumbosacral enlargements of cats and monkeys. Somatosens Res 1:131-149.

Churchill JD, Tharp JA, Wellman CL, Sengelaub DR, Garraghty PE (2004) Morphological correlates of injury-induced reorganization in primate somatosensory cortex. BMC Neurosci 5:43.

Cooper BY, Glendinning DS, Vierck CJ Jr (1993) Finger movement deficits in the stumptail macaque following lesions of the fasciculus cuneatus. Somatosens Mot Res 10:17-29.

Cusick CG, Steindler DA, Kaas JH (1985) Corticocortical and collateral thalamocortical connections of postcentral somatosensory cortical areas in squirrel monkeys: a double-labeling study with radiolabeled wheatgerm agglutinin and wheatgerm agglutinin conjugated to horseradish peroxidase. Somatosens Res 3:1-31.

Darian-Smith C, Brown S (2000) Functional changes at periphery and cortex following dorsal root lesions in adult monkeys. Nat Neurosci 3:476-481

Darian-Smith C, Ciferri MM (2005) Loss and recovery of voluntary hand movements in the macaque following a cervical dorsal rhizotomy. J Comp Neurol 491:27-45.

Dreyer DA, Schneider RJ, Metz CB, Whitsel BL (1974) Differential contributions of spinal pathways to body representation in postcentral gyrus of Macaca mulatta. J Neurophysiol 37:119-145.

Dykes RW, Craig AD (1998) Control of size and excitability of mechanosensory receptive fields in dorsal column nuclei by homolateral dorsal horn neurons. J Neurophysiol 80:120-129.

Florence SL, Kaas JH (1995) Large-scale reorganization at multiple levels of the somatosensory pathway follows therapeutic amputation of the hand in monkeys. J Neurosci 15:8083-8095.

Florence SL, Wall JT, Kaas JH (1988) The somatotopic pattern of afferent projections from the digits to the spinal cord and cuneate nucleus in macaque monkeys. Brain Res 452:388-392.

Florence SL, Wall JT, Kaas JH (1989) Somatotopic organization of inputs from the hand to the spinal gray and cuneate nucleus of monkeys with observations on the cuneate nucleus of humans. J Comp Neurol 286:48-70.

Florence SL, Wall JT, Kaas JH (1991) Central projections from the skin of the hand in squirrel monkeys. J Comp Neurol 311:563-578.

Florence SL, Garraghty PE, Wall JT, Kaas JH (1994) Sensory afferent projections and area $3 \mathrm{~b}$ somatotopy following median nerve cut and repair in macaque monkeys. Cereb Cortex 4:391-407.

Florence SL, Jain N, Pospichal MW, Beck PD, Sly DL, Kaas JH (1996) Cen- 
tral reorganization of sensory pathways following peripheral nerve regeneration in fetal monkeys. Nature 381:69-71.

Florence SL, Taub HB, Kaas JH (1998) Large-scale sprouting of cortical connections after peripheral injury in adult macaque monkeys. Science 282:1117-1121.

Gallyas F (1979) Silver staining of myelin by means of physical development. Neurol Res 1:203-209.

Garraghty PE, Florence SL, Kaas JH (1990) Ablations of areas 3a and 3b of monkey somatosensory cortex abolish cutaneous responsivity in area 1 . Brain Res 528:165-169.

Garraghty PE, LaChica EA, Kaas JH (1991) Injury-induced reorganization of somatosensory cortex is accompanied by reductions in GABA staining. Somatosens Mot Res 8:347-354.

Garraghty PE, Arnold LL, Wellman CL, Mowery TM (2006) Receptor autoradiographic correlates of deafferentation-induced reorganization in adult primate somatosensory cortex. J Comp Neurol 497:636-645.

Gharbawie OA, Stepniewska I, Kaas JH (2011) Cortical connections of functional zones in posterior parietal cortex and frontal cortex motor regions in new world monkeys. Cereb Cortex. Advance online publication. Retrieved August 11, 2001. doi:10.1093/cercor/bhq260.

Graziano A, Jones EG (2009) Early withdrawal of axons from higher centers in response to peripheral somatosensory denervation. J Neurosci 29:3738-3748.

Jain N, Catania KC, Kaas JH (1997) Deactivation and reactivation of somatosensory cortex after dorsal spinal cord injury. Nature 386:495-498.

Jain N, Florence SL, Kaas JH (1998) Reorganization of somatosensory cortex after nerve and spinal cord injury. News Physiol Sci 13:143-149.

Jain N, Florence SL, Qi HX, Kaas JH (2000) Growth of new brainstem connections in adult monkeys with massive sensory loss. Proc Natl Acad Sci U S A 97:5546-5550.

Jain N, Qi HX, Collins CE, Kaas JH (2008) Large-scale reorganization in the somatosensory cortex and thalamus after sensory loss in macaque monkeys. J Neurosci 28:11042-11060.

Jones EG (1993) GABAergic neurons and their role in cortical plasticity in primates. Cereb Cortex 3:361-372.

Juliano SL, Friedman DP, Eslin DE (1990) Corticocortical connections predict patches of stimulus-evoked metabolic activity in monkey somatosensory cortex. J Comp Neurol 298:23-39.

Kaas JH (2001) The mutable brain. New York: Harwood Academic Publishers.

Kaas JH, Qi HX, Burish MJ, Gharbawie OA, Onifer SM, Massey JM (2008) Cortical and subcortical plasticity in the brains of humans, primates, and rats after damage to sensory afferents in the dorsal columns of the spinal cord. Exp Neurol 209:407-416.

Leonard CM, Glendinning DS, Wilfong T, Cooper BY, Vierck CJ Jr (1992) Alterations of natural hand movements after interruption of fasciculus cuneatus in the macaque. Somatosens Mot Res 9:75-89.

Merzenich MM, Kaas JH, Sur M, Lin CS (1978) Double representation of the body surface within cytoarchitectonic areas $3 \mathrm{~b}$ and 1 in "SI" in the owl monkey (Aotus trivirgatus). J Comp Neurol 181:41-73.

Merzenich MM, Kaas JH, Wall J, Nelson RJ, Sur M, Felleman D (1983a) Topographic reorganization of somatosensory cortical areas $3 \mathrm{~b}$ and 1 in adult monkeys following restricted deafferentation. Neuroscience $8: 33-55$.

Merzenich MM, Kaas JH, Wall JT, Sur M, Nelson RJ, Felleman DJ (1983b)
Progression of change following median nerve section in the cortical representation of the hand in areas $3 \mathrm{~b}$ and 1 in adult owl and squirrel monkeys. Neuroscience 10:639-665.

Muellbacher W, Hallett M (2006) Reprogramming surviving motor cortex after stroke. In: Reprogramming the cerebral cortex: plasticity following central and peripheral lesions (Lomber S, Eggermont J, eds), pp 257-272. New York: Oxford UP.

Nudo RJ, Barbay S, Kleim JA (2000) Role of neuroplasticity in functional recovery after stroke. In: Cerebral reorganization of function after brain damage (Levin SL, Grafman J, eds), pp 168-197. New York: Oxford UP.

Olausson HW, Cole J, Vallbo A, McGlone F, Elam M, Krämer HH, Rylander K, Wessberg J, Bushnell MC (2008) Unmyelinated tactile afferents have opposite effects on insular and somatosensory cortical processing. Neurosci Lett 436:128-132.

Pons TP, Garraghty PE, Ommaya AK, Kaas JH, Taub E, Mishkin M (1991) Massive cortical reorganization after sensory deafferentation in adult macaques. Science 252:1857-1860.

Qi HX, Kaas JH (2006) Organization of primary afferent projections to the gracile nucleus of the dorsal column system of primates. J Comp Neurol 499:183-217.

Qi HX, Jain N, Collins CE, Lyon DC, Kaas JH (2010) Functional organization of motor cortex of adult macaque monkeys is altered by sensory loss in infancy. Proc Natl Acad Sci U S A 107:3192-3197.

Ramachandran VS (1993) Behavioral and magnetoencephalographic correlates of plasticity in the adult human brain. Proc Natl Acad Sci U S A 90:10413-10420.

Ramachandran VS, Rogers-Ramachandran D, Stewart M (1992) Perceptual correlates of massive cortical reorganization. Science 258:1159-1160.

Randolph M, Semmes J (1974) Behavioral consequences of selective subtotal ablations in the postcentral gyrus of Macaca mulatta. Brain Res 70:55-70.

Rustioni A, Hayes NL, O’Neill S (1979) Dorsal column nuclei and ascending spinal afferents in macaques. Brain 102:95-125.

Schroeder CE, Seto S, Arezzo JC, Garraghty PE (1995) Electrophysiological evidence for overlapping dominant and latent inputs to somatosensory cortex in squirrel monkeys. J Neurophysiol 74:722-732.

Strata F, Coq JO, Kaas JH (2003) The chemo- and somatotopic architecture of the Galago cuneate and gracile nuclei. Neuroscience 116:831-850.

Sur M, Nelson RJ, Kaas JH (1982) Representations of the body surface in cortical areas $3 \mathrm{~b}$ and 1 of squirrel monkeys: comparisons with other primates. J Comp Neurol 211:177-192.

Tang XN, Berman AE, Swanson RA, Yenari MA (2010) Digitally quantifying cerebral hemorrhage using Photoshop and Image J. J Neurosci Methods 190:240-243.

Vogt BA, Pandya DN (1978) Cortico-cortical connections of somatic sensory cortex (areas 3,1 and 2) in the rhesus monkey. J Comp Neurol 177:179-191.

Willis WD, Coggeshall RE (2004) Sensory pathways in the dorsal funiculus. In: Sensory mechanisms of the spinal cord: ascending sensory tracts and their descending control (Willis WD, Coggeshall RE, eds), pp 597-664. New York: Kluwer Academy/Plenum Publisher.

Wong-Riley M (1979) Changes in the visual system of monocularly sutured or enucleated cats demonstrable with cytochrome oxidase histochemistry. Brain Res 171:11-28. 